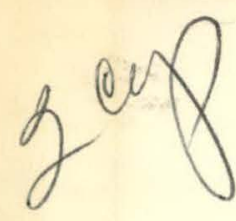

BASICANDAPPLIEDRESEARCH IN THE EARTH SCIENCES

\title{
SEISMIC SPECTRUM SCALING OF UNDERGROUND DETONATIONS
}

BY

RICHARD A. MUELLER

JOHN R. MURPHY

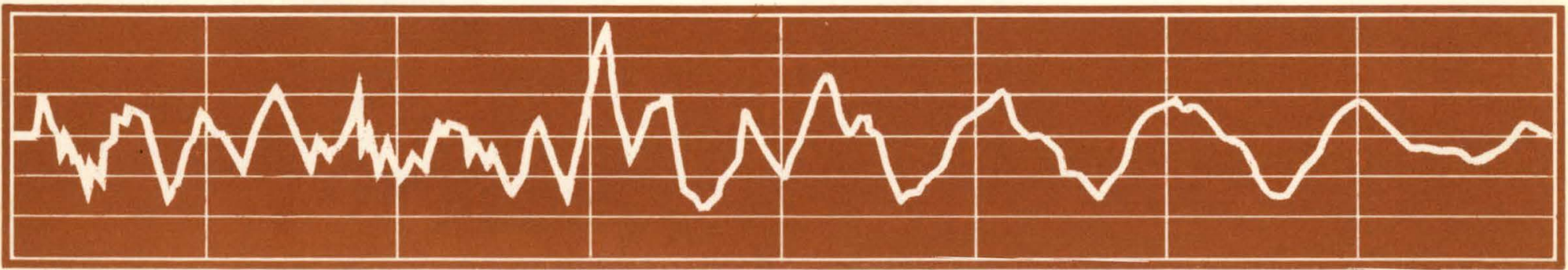

E N V I R O N M E N T A L RE S E A R C H

$\begin{array}{llllllllllllllllllllll}C & 0 & R & P & 0 & R & A & T & \text { I } & 0 & N\end{array}$

a s u b s i d i a r y

$0 \mathrm{f}$

c o m p u t e i 


\section{DISCLAIMER}

This report was prepared as an account of work sponsored by an agency of the United States Government. Neither the United States Government nor any agency Thereof, nor any of their employees, makes any warranty, express or implied, or assumes any legal liability or responsibility for the accuracy, completeness, or usefulness of any information, apparatus, product, or process disclosed, or represents that its use would not infringe privately owned rights. Reference herein to any specific commercial product, process, or service by trade name, trademark, manufacturer, or otherwise does not necessarily constitute or imply its endorsement, recommendation, or favoring by the United States Government or any agency thereof. The views and opinions of authors expressed herein do not necessarily state or reflect those of the United States Government or any agency thereof. 


\section{DISCLAIMER}

Portions of this document may be illegible in electronic image products. Images are produced from the best available original document. 
SEISMIC SPECTRUM SCALING

$\mathrm{OF}$

\section{UNDERGROUND DETONATIONS}

\section{by}

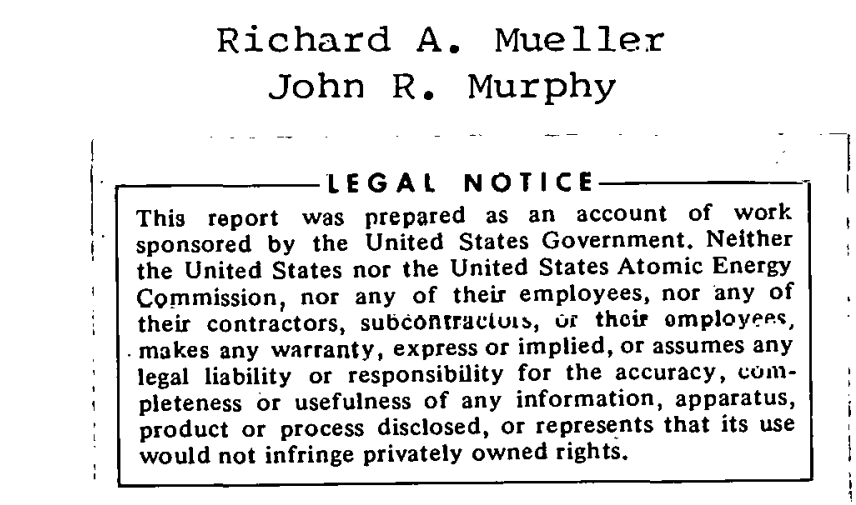

Environmental Research Corporation

2769 South Highland Drive

Las Vegas, Nevada

Prepared under

Contract AT(29-2)-1163

for the

Nevada Operations Office

U.S. Atomic Energy Commission 
TABLE OF CONTENTS

Chapter

$\underline{\text { Page }}$

ABSTRACT ....................... v

1 INTRODUCTION ..................... 1-1

$2 \quad$ THEORY ........................... 2-1

3 APPLICATIONS OF THEORY................. 3-1

4 EXTENSION OF THE SPECTRUM SCALING THEORY TO PEAK GROUND MOTION SCALING.............. 4-1

5 SUMMARY AND RECOMMENDATIONS............. 5-1

REFERENCES ........................ R- 1

\section{LIST OF ILLUSTRATIONS}

Figure

Page

2-1 Pressure Profile at the Elastic Radius....... 2-3

2-2 Plot of $\left(\omega_{o} / \omega_{m}\right)$ versus $k \ldots \ldots \ldots \ldots \ldots \ldots \ldots . \ldots \ldots$

$2-3 \quad$ Plot of $k$ versus $\left(\omega_{\mathrm{m}} / a\right) \ldots \ldots \ldots \ldots \ldots \ldots \ldots \ldots \ldots$

2.-4 Plot of Elastic Radius/Cube-Root of Yield

Versus Density Times Depth of Builal for

Various Shots (Circles are Events Detonated

in Tuff or Rhyolite) ...................2-13

3-1 Comparison of Theoretical and Empirical Yield Scaling Exponents...................... 3-3

3-2 Comparison of Theoretical and Empirical Yield Scaling Exponents Derived from Eight Single Station PSRV Analysis................. 3-5 
3-3 Theoretical Yield Scaling Exponents for

Set Depth of Burial Events............ 3-7

3-4 Theoretical Depth Scaling Exponents for

Set Yield Events.................. 3-8

3-5 Comparison of Knickerbocker, Scaled

Knickerbocker and Cabriolet PSRV (5\%

Damping) at Allamo (Horizontal Component).... 3-10

3-6 Comparison of Rex, Scaled Rex and Cabriolet

PSRV (5\% Damping) at SE-6 (Horizontal

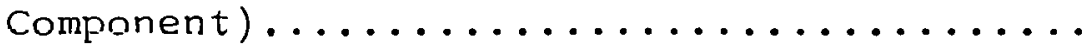

3-7 Comparison of Knickerbocker, Scaled

Knickerbocker and Schooner PSRV (5\% Damping)

at Tonopah Church (Horizontal Component)....

3-8 Comparison of Rex, Scaled Rex and Schooner PSRV (5\% Damping) at SE-6 (Horizontal

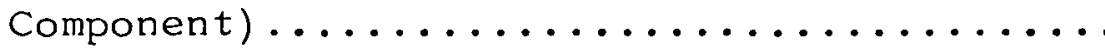

3-9 Comparison of PSRV (5\% Damping) from

Cabriolet with PSRV from Knickerbocker and Rex Theoretically Scaled, to Equivalent

Physical Parameters Except at a Contained

Depth of Burial ( $S D=400)$, Station $S E-6$,

Horizontal Component................

3-10 Comparison of PSRV (5\% Damping) from

Schooner with PSRV from Knickerbocker and

Rex Theoreticaled Scaled to Equivalent

Physical Parameters Except at a Contained

Depth of Burial $(\mathrm{SD}=400)$, Station SE-6,

Horizontal Component................ 3-16

3-11 Comparison of Event A, Event A Scaled and Greeley PSRV (5\% Damping) at SE-6

(Horizontal Component). 


\section{LIST OF ILLUSTRATIONS (CONTINUED)}

Figure

$\underline{\text { Page }}$

3-12 Comparison of Event A, Event A Scaled and Cabriolet PSRV (5\% Damping) at SE-6

(Horizontal Component) ................ 3-19

4-1 Comparison of Observed Gașbuggy and Pre-

dicted Peak Accelerations at Hard Rock and

Alluvium stations..................... 4-5

4-2 Comparison of Observed Gasbuggy and Pre-

dicted Peak Particle Displacements at Hard

Rock and Alluvium Stations............. 4-7

4-3 Comparison of Gasbuggy and Predicted PSRV

(5\% Damping) at $10 \mathrm{~km} \ldots \ldots . . \ldots \ldots . . . . . .4$

4-4 Comparison of Gasbuggy and Predicted PSRV

(5\% Damping) at $80 \mathrm{~km} \ldots \ldots \ldots . \ldots . \ldots . \ldots . . .4-10$

4-5 Comparison of Observed Gasbuggy and Pre-

dicted Peak Particle Velocities at Hard

Rock and Alluvium Stations.............4-11

4-6 Comparison of Observed Rulison Peak

Particle Accelerations with the Accelera-

tions Theoretically and Empirically Scaled

from Gasbuggy..................... 4-12

4-7 Comparison of Observed Rulison Peak

Farticlc Displarements with the Displacements Theoretically and Empirically Scaled

from Gasbuggy.................... 4-13 


\section{ABSTRACT}

A general scaling theory of ground motions resulting from underground nuclear detonations. is developed from the solution of the wave equation with appropriate boundary conditions. The analysis shows that in addition to yield, the device depth of burial and the medium play a significant role in the scaling of seismic signals. The theory predicts an increase in the dominant frequency and ground accelerations with an increase of device depth of burial. This phenomenon is verified by observations from excavation events and overburied events. For events buried sufficiently deep for containment, the scaling theory predicts frequency dependent yield exponents (ranging from 0.90 at low frequencies to 0.45 at high frequencies) which are in good agreement with empirical relations statistically derived from a large number of events. 
CHAPTER 1

\section{INTRODUCTION}

The subject of this paper is the scaling of the amplitude spectra of seismic waves from underground detonations. A model and associated analysis are presented which quantitatively account for differences in spectra from different events by considering differences in shot point parameters. Scaling is, in general, frequency, yield, depth of burial and medium dependent. For the particular case of shots buried at a typical contained depth, in a specific medium it is found that the yield scaling exponent decreases as the frequency increases, which is in agreement with experimental evidence.

The situation to be considered is that of a source, representing the explosion, a transmission function representing the transfer of energy from the source to the receiver and the receiver itself. The model. used to represent the source is that of a spherically symmetric pressure function acting at an elastic radius, $i . e$. , the radius at which the medium behaves elastically. The solution to this mathematical boundary value problem has been developed by several authors. 
Now, consider a seismogram recording at a recording station, say some $200 \mathrm{~km}$ away from the source. Such a seismogram is a complex signal composed of many different arrivals and wave types. The amplitude spectrum at this distant station can be functionally represented by a transfer function representing the earth from the source to the recording station multiplied by the source input. This transfer function is very complex. It represents many different travel paths, wave mode conversions, differential scattering, and amplification effects from the source to the receiver. The analytical evaluation of this function would be a monumental endeavor. However, if we are considering observations at some common distant station for two proximate events, the transfer functions are approximately equal and the ratio of the seismic spectra at the distant station is equivalent to the ratio close-in. The transfer function has thus been conveniently eliminated.

At $t$ his point, the stage has been set and the next step is the theoretical evaluation of the scaling scheme. 


\section{CHAPTER 2}

\section{THEORY}

The solution to the spherically symmetric problem of a forcing function acting in an infinite homogeneous medium has been developed by Sharpe (1942) in the time domain and by Latter, et al (1959) in the frequency domain. The solution in the frequency domain. is easily derived from the wave equation and Hooke's law. It is assumed that there exists a spherical surface surrounding the detonation point outside of which infinitesimal strain theory is applicable. Denoting the radius of this sphere as the elastic radius rel, then for radial distances $R \geqslant r_{e l}$, the equation of motion reduces to the one-dimensional wave equation in the displacement potential, $\varphi$,

$$
\frac{\partial^{2} \varphi}{\partial r^{2}}=\frac{1}{c^{2}} \frac{\partial^{2} \varphi}{\partial t^{2}}
$$

which has the solution $\varphi=\varphi\left[\cdot t-\left(x-x_{e} l\right) / c\right]$ for outgoing spher1cal waves. The displacement, $Z$, is related to the displacement potential by

$$
\mathcal{Z}=\frac{\partial(\varphi / r)}{\partial r}
$$

Hooke's law gives the relationship between the pressure, $p(t)$, and displacement at $r_{e l}$ as 


$$
p(t)=-(\lambda+2 \mu)\left(\frac{\partial Z}{\partial r}\right)-2 \lambda\left(\frac{Z}{r}\right)
$$

where $\lambda$ and $\mu$ are Lamss constants.

Substituting equation (2) in (3) for $z$, taking the Fourier transform and substituting the Fourier transform of $\varphi$ in terms of the Fourier transform of $Z$ in the resulting equation gives

$$
\hat{z}(\omega)=\frac{\hat{p} r_{e l}}{4 \mu}\left(\frac{1}{R^{2}}+\frac{i \omega}{R c}\right) \frac{c^{2}}{\left(\omega_{0}^{2}+i \omega_{\Omega} \omega-\beta \omega^{2}\right)}
$$

where $\hat{Z}$ is the Fourier transform of the displacement at $R$, $\hat{\mathrm{p}}$ is the Fourier transform of the pressure function at $\mathrm{r}_{\mathrm{el}}$, $\omega$ is the modulus of rigidity, $c$ is the compressional velocity, $\beta=\frac{\lambda+2 \mu}{4 \mu}$, $\omega$ is the angular frequency $(2 \pi f)$ and $\omega_{\mathrm{O}}=c / \mathrm{r}_{\mathrm{el}}$ :

The amplitude spectrum at some distant point is equal to the input into the ground at $R$, given by equation (4), times some transfer function $T(\omega)$, i.e.

$$
|\hat{z}|_{\text {Dist }}=T(\omega)|\hat{z}|_{R}
$$

Considering observations at some common distant station for two proximate detonations and assuming the transfer function to be linear, we may write 


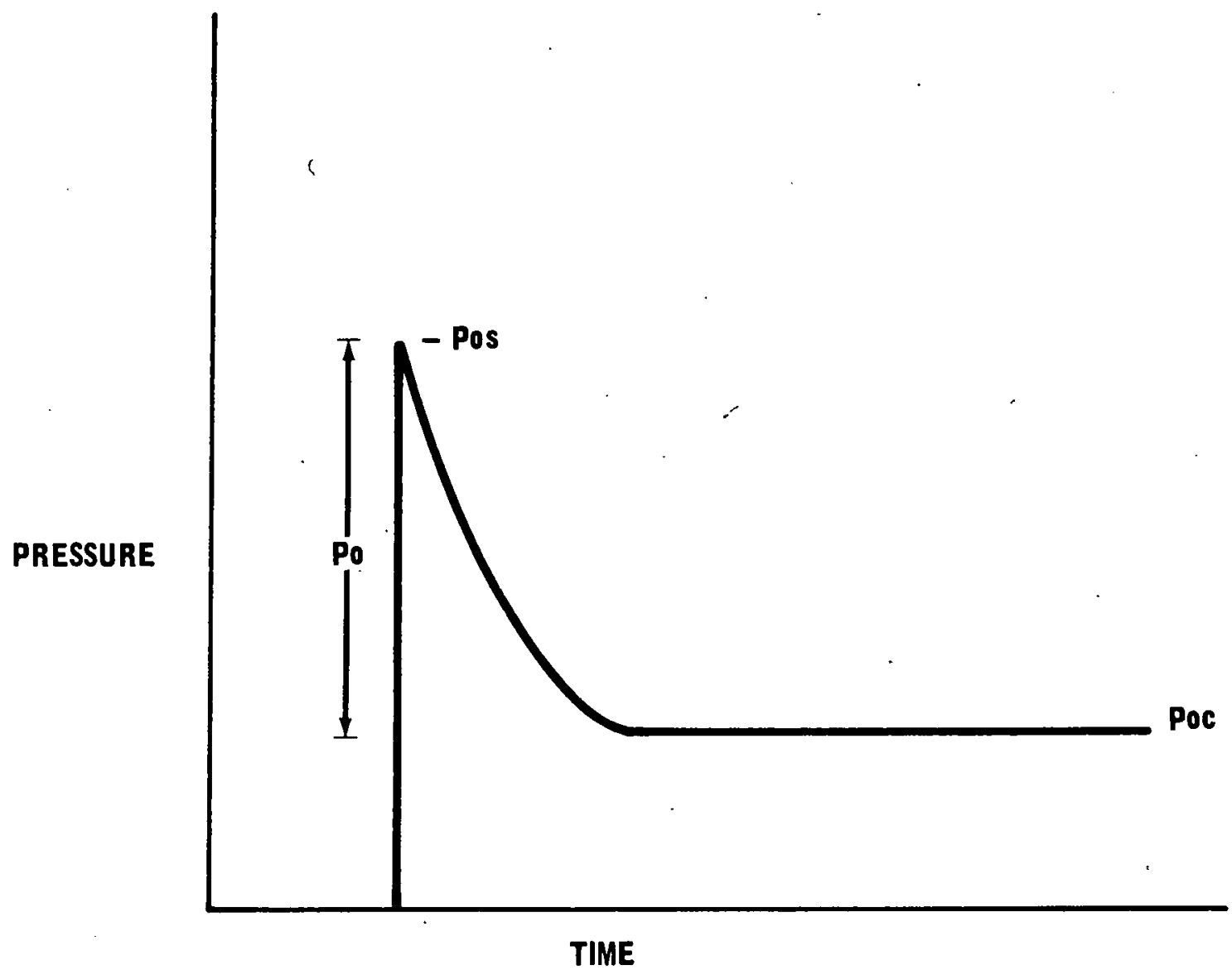

Figure 2-1. Pressure Profile at the Elastic Radius 


$$
\frac{\left|\hat{z}_{1}\right|_{\text {Dist }}}{\left|\hat{z}_{2}\right|_{\text {Dist }}}=\frac{T(\omega)\left|\hat{z}_{1}\right|_{R}}{T(\omega)\left|\hat{z}_{2}\right|_{R}}
$$

where the transfer function is taken to be the same since the propagation paths are approximately equal. Thus, for two shots with different shot point parameters; we have from equations (6) and (4),

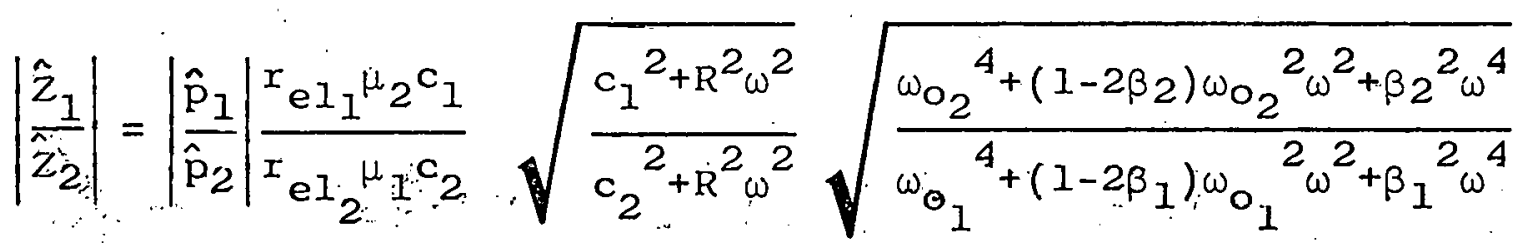

The pressure function at the elastic radius is composed of the shock wave plus the stress produced by the cavity expansion (Figure 2-1). This form of the forcing function is deduced from free-field observations of events such as Salmon (Perret (1968)), Hardhat (Perret (1965)) and Shoal (Weart (1965)). This pressure profile may be represented by a funstion of the form

$$
p(t)=\left(p_{O} e^{-\alpha t}+p_{O C}\right) H(t)
$$

where $\mathrm{p}_{\mathrm{o}}+\mathrm{p}_{\mathrm{oc}}=\mathrm{p}_{\mathrm{os}}$ is the peak pressure and $\mathrm{p}_{\mathrm{OC}}$ is the steady state stress, $\alpha$ is the decay constant; and $H(t)$ is the unit step function. 
The Fourier transform of $p(t)$ is

$$
\hat{p}(\omega)=\frac{\left(P_{\text {OS }}-P_{\text {OC }}\right)}{\alpha+i \omega}+\frac{p_{\text {oC }}}{i \omega}
$$

and its amplitude

$$
|\hat{p}(\omega)|=\frac{1}{\omega} \sqrt{\frac{\nu^{2} p_{o s}^{2}+p_{o c}^{2} \alpha^{2}}{\alpha^{2}+\omega^{2}}}
$$

Then, from equations (4) and (6) the important full scaling equation relating the amplitude spectra of two events becomes:

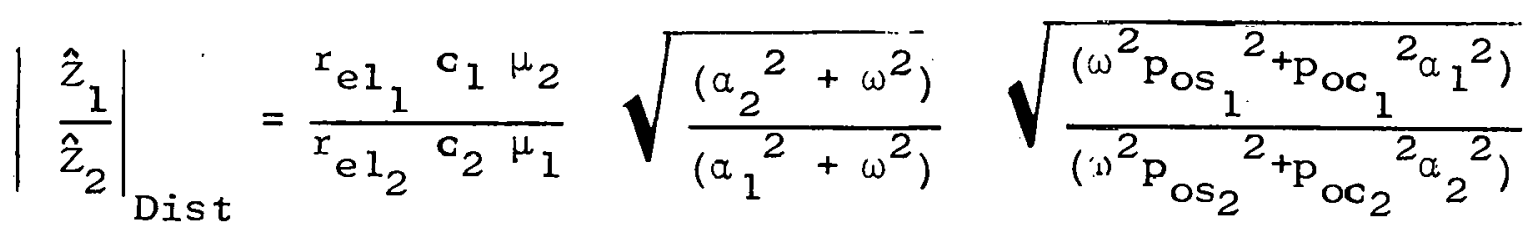

$$
\begin{aligned}
& \times \sqrt{\frac{\mathrm{c}_{1}^{2}+\mathrm{R}^{2} \omega^{2}}{\mathrm{c}_{2}^{2}+\mathrm{R}^{2} \omega^{2}}} \times \sqrt{\frac{\beta_{2}^{2} \omega^{4}+\left(1-2 \beta_{2}\right) \omega_{o_{2}}^{2} \omega^{2}+\omega_{o_{2}}^{4}}{\beta_{1}^{2} \omega^{4}+\left(1-2 \beta_{1}\right) \omega_{o_{1}}^{2} \omega^{2}+\omega_{o_{1}}^{4}}}
\end{aligned}
$$

where $R \geqslant$ the larger of the two elastic radii.

$$
\text { Thus, iff }\left|z_{2}\right|_{\text {Dist }} \text { is known, equation (11) determines }
$$
$\left|z_{1}\right|_{\text {Dist }}$. The parameters $o, \mu$ and $\beta$ are measureable characteristics of the medium. Thus, in order to evaluate equation (11), a functional relationship of $r_{e l}, a, p_{o s}$ and $p_{o c} w i t h$ yield, depth of burial and medium must be determined. 
The steady state cavity expansion stress at the elastic radius, Poc, can be determined from an incompressible model and Hooke's law. The permanent displacement at $r_{e l}$ in an incompressible material where the cavity grows from an initial vaporization radius $r_{v}$ to a final cavity radius of $r_{c}$ is

$$
z_{p}=\left[r_{e 1}{ }^{3}+r_{c}^{3}-r_{v}^{3}\right]^{1 / 3}-r_{e l}
$$

Now, since $r_{v}{ }^{3}$ is very small compared to $\left(r_{e l}{ }^{3}+r_{c}{ }^{3}\right)$ it may be neglected. Since $r_{c} / r_{e l} \ll 1$, we may binomially expand the first term on the right side of equation (12) to $r_{e l}\left[1+1 / 3\left(r_{c} / r_{e l}\right)^{3}\right]$ to give

$$
\left.z_{p}\right|_{r_{e l}}=\frac{1}{3} \frac{r_{c}^{3}}{r_{e l}^{2}}
$$

Equation (13) holds for any $r>r_{c}$, i.e.,

$$
z_{p}^{\prime} r_{r}=\frac{1}{3} \frac{r_{c}{ }^{3}}{r^{2}}
$$

Hooke's law (equation 3) gives the relation between stress and displacement at $r$. Substituting equation (14) into Hooke's relation at $r_{e l}$ gives

$$
\left.p_{x c}\right|_{r_{e l}}=\frac{4 \mu}{3}\left(\frac{r_{c}}{r_{e l}}\right)^{3}
$$


Thus, equation (15) gives a relationship.between the parameter Poc and final cavity radius for an incompressible material.

The decay time of the pressure function at the elastic radius, which is the inverse of $a$, is taken to scale as the elastic radius. Thus $a=k \omega_{0}$, where $k$ is a proportionality constant. The limiting pressure, Pos, was found by Mueller (1969) to be slightly larger than the overburden pressure. A factor of 1.5 appears appropriate. In the inelastic region, peak shock pressure, Pos, is taken to follow a power law of the form

$$
\mathrm{p}_{\mathrm{os}}=\frac{\mathrm{A} w^{\mathrm{m}}}{\mathrm{r}^{\mathrm{n}}}
$$

where $A$ is a constant; $W$ is yiell and $I$ is distance. For cube root scaling to hold for the vaporization cavity radius or any radius associated with a transition pressure in the inelastic region of a particular medium, $m=n / 3$ and the relationship between the elastic radii is

$$
\frac{\mathrm{r}_{\mathrm{el}}}{\mathrm{r}_{\mathrm{el}}}=\left(\frac{\mathrm{W}_{2}}{\mathrm{~W}_{2}}\right)^{1 / 3}\left(\frac{\mathrm{P}_{\mathrm{ob}}}{\mathrm{P}_{\mathrm{ob}}}\right)^{1 / \mathrm{n}}
$$


and

$$
\frac{{ }^{s} o_{1}}{\omega \mathrm{o}_{2}}=\left(\frac{\mathrm{w}_{2}}{\mathrm{w}_{1}}\right)^{1 / 3}\left(\frac{\mathrm{P}_{\mathrm{ob}_{1}}}{\mathrm{P}_{\mathrm{ob}_{2}}}\right)^{1 / \mathrm{n}}
$$

where $\mathrm{p}_{\mathrm{ob}}$ is the overburden pressure.

For shots buried in different media, the constant A appearing in equation (16) will be different. The constant $n$ is taken to be approximately the same for all media. Sauer (1964), who correlated inelastic motions from shots in different media, has shown that this procedure is valid. Thus, the general scaling formula for $\mathrm{r}_{\mathrm{el}}$ is

$$
\frac{\mathrm{r}_{\mathrm{e} l_{1}}}{\mathrm{r}_{\mathrm{el}}}=\left(\frac{\mathrm{A}_{1}}{\mathrm{~A}_{2}}\right)^{1 / \mathrm{n}}\left(\frac{\mathrm{W}_{1}}{\mathrm{~W}_{2}}\right)^{1 / 3}\left(\frac{\rho_{2} \mathrm{~h}_{2}}{\rho_{1} \mathrm{~h}_{1}}\right)^{1 / \mathrm{n}}
$$

where $\rho$ and $h$ are the medium density and device depth of burial respectively.

In order to evaluate the scaling equation (11), an absolute determination of the elastic radius within a self-consistent method is necessary. This can be effected by considering the amplitude spectra of the compressional wave in the far field resulting from the shock wave. The assumption underlying this method is that the peak of the shock wave amplitude spectrum is much larger and/or sufficiently separated from the peak of the 
cavity expansion pressure wave amplitude spectrum to allow a separate analysis. This assumption is borne out by experiment. From equation (4), the shock wave velocity amplitude spectrum in the far field $(R>>c / \omega)$ can be obtained as (with the substitution $\left.a=k \omega_{0}\right)$

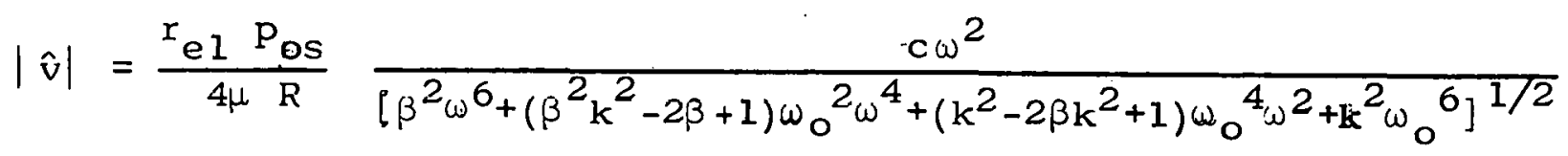

which has a maximum at $\omega_{m}$ given by the dimensionless equation:

$$
\beta^{2}\left(\frac{\omega_{m}}{\omega_{o}}\right)^{6}-\left(k^{2}-2 \beta k^{2}+1\right)\left(\frac{\omega_{m}}{\omega_{o}}\right)^{2}-2 k^{2}=0
$$

Figure $2-2$ shows a plot of $\left(\omega_{m} / \omega_{0}\right)$ versus $k$ for various values of Poisson's ratio $(\nu)$. By substituting $\omega_{0}=a / k$ in equation (21), one obtains the relationship between $k$ and $\left(\omega_{\mathrm{m}} / \alpha\right)$ as

$$
\beta^{2}\left(\frac{\omega_{m}}{\alpha}\right)^{6} k^{4}+(2 \beta-1)\left(\frac{\omega_{m}}{\alpha}\right)^{2} k^{2}-\left(\frac{\omega_{m}}{a}\right)^{2}-2=0
$$

Figure 2-3 shows a plot of $k$ versus $\omega_{\mathrm{m}} / \alpha$ for various values of Poisson's ratio. The parameter $k$ is assumed to be constant for a particular shot point medium. Given the value of $\omega_{\mathrm{m}}$ (angular frequency of maximum amplitude of the compressional wave) from distant seismic data, and $u$ (inverse of the decay time of the shock wave) from those instances (such as Salmon, Rainier, Gasbuggy) 


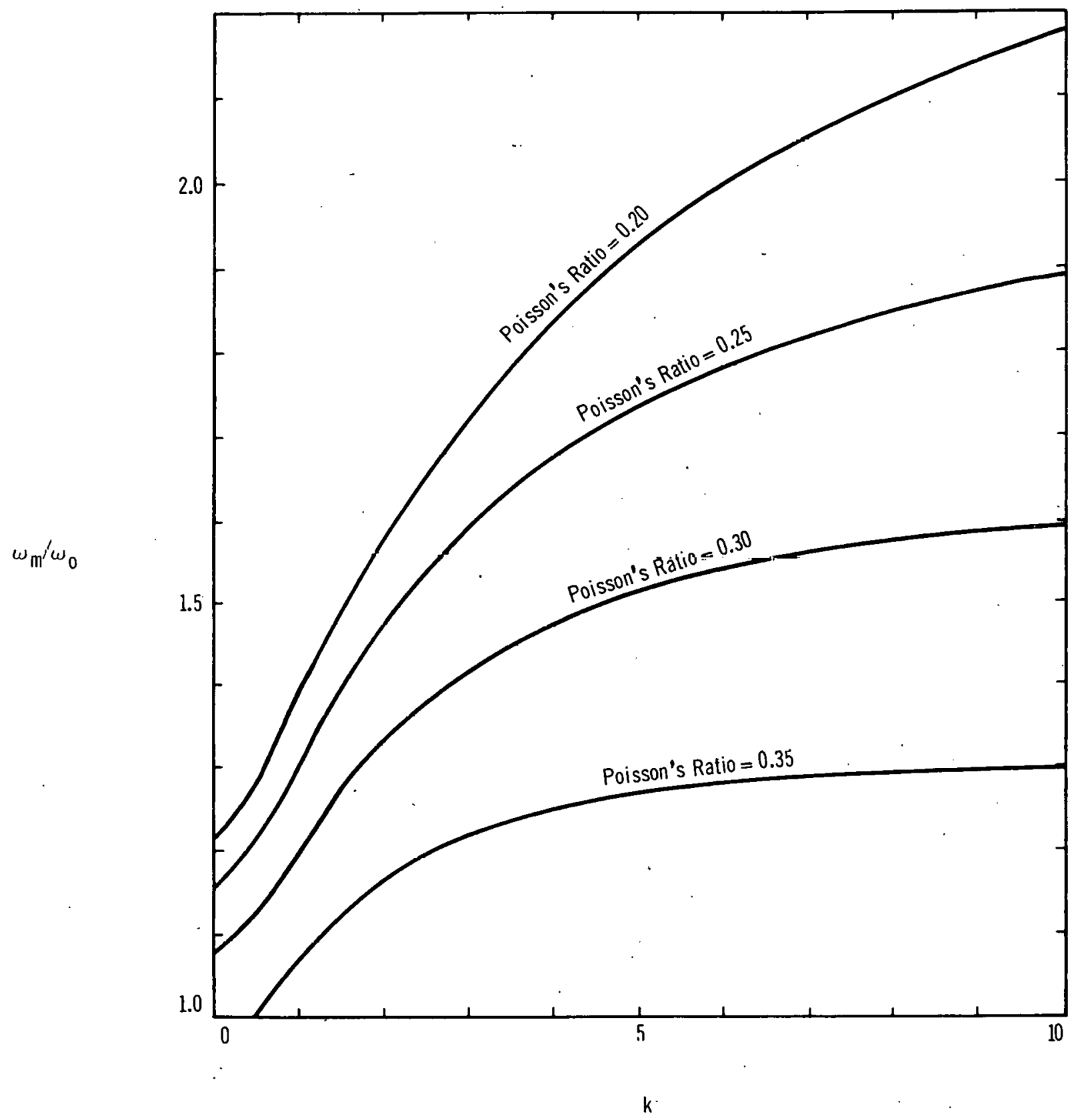

Figure 2-2. Plot of $\left(\omega_{0} / \omega_{m}\right)$ versus $k$ 


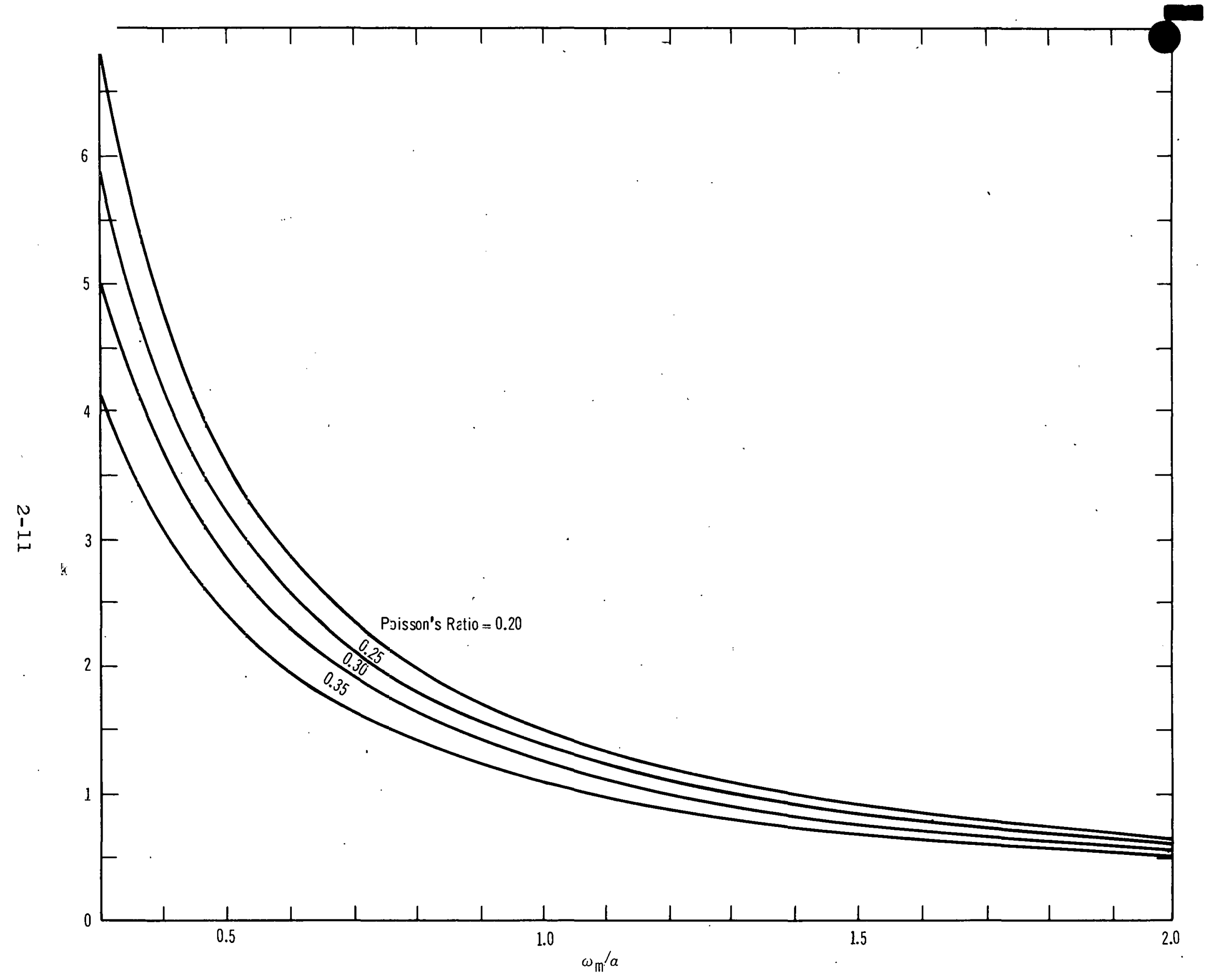

Figure 2-3. Plot of $k$ versus $\left(\omega_{\mathrm{m}} / \alpha\right)$ 
where it has been measured close-in, the ratio $\left(\omega_{m} / a\right)$ and Poisson's ratio yield $k$ through Figure 2-3 and $\omega_{0}\left(=c / r_{e l}\right)$ through Figure $2-2$. Approximate $k$ values determined for various media are: tuff, $k=1.5 ;$ rhyolite; $k=2.0 ;$ shale and sandstone, $k=2.4 ;$ salt; $k=4.5$.

These results are utilized in investigating the consequences of equation (19). By considering event 2 to be a calibration shot, equation (19) can be rearranged as

$$
\frac{r_{e l}}{w^{1 / 3}}=\frac{G \cdot A^{1 / n}}{(\rho h)^{1 / n}}
$$

where $G$ is a constant and $A$ is only dependent on shot point medium. The object now is to plot $r_{e l} / W^{1 / 3}$ for many events detonated in similar media (A-constant) in order to obtain the exponent $\mathrm{n}$ and also a calibration base. Events in other media can then be compared to this base which will determine relative values of $A$, i.e., relative peak shock pressures in different media. This result will be utilized in the full scaling scheme.

Events detonated in tuff and rhyolite. (t-r), within the NTS Testing grounds, are used for the above base. The elastic radii are determined from $\omega_{m}$, Poisson's ratio, $c, k$, and Figure 2-3 relating $\omega_{\mathrm{o}}\left(=\mathrm{c} / \mathrm{r}_{\mathrm{el}}\right)$ to $\omega_{\mathrm{m}}$. Figure $2-4$ shows $a$ plot of $\log \left(r_{e l} / \omega^{1 / 3}\right)$. A value of $n=2.4$ fits the data. 


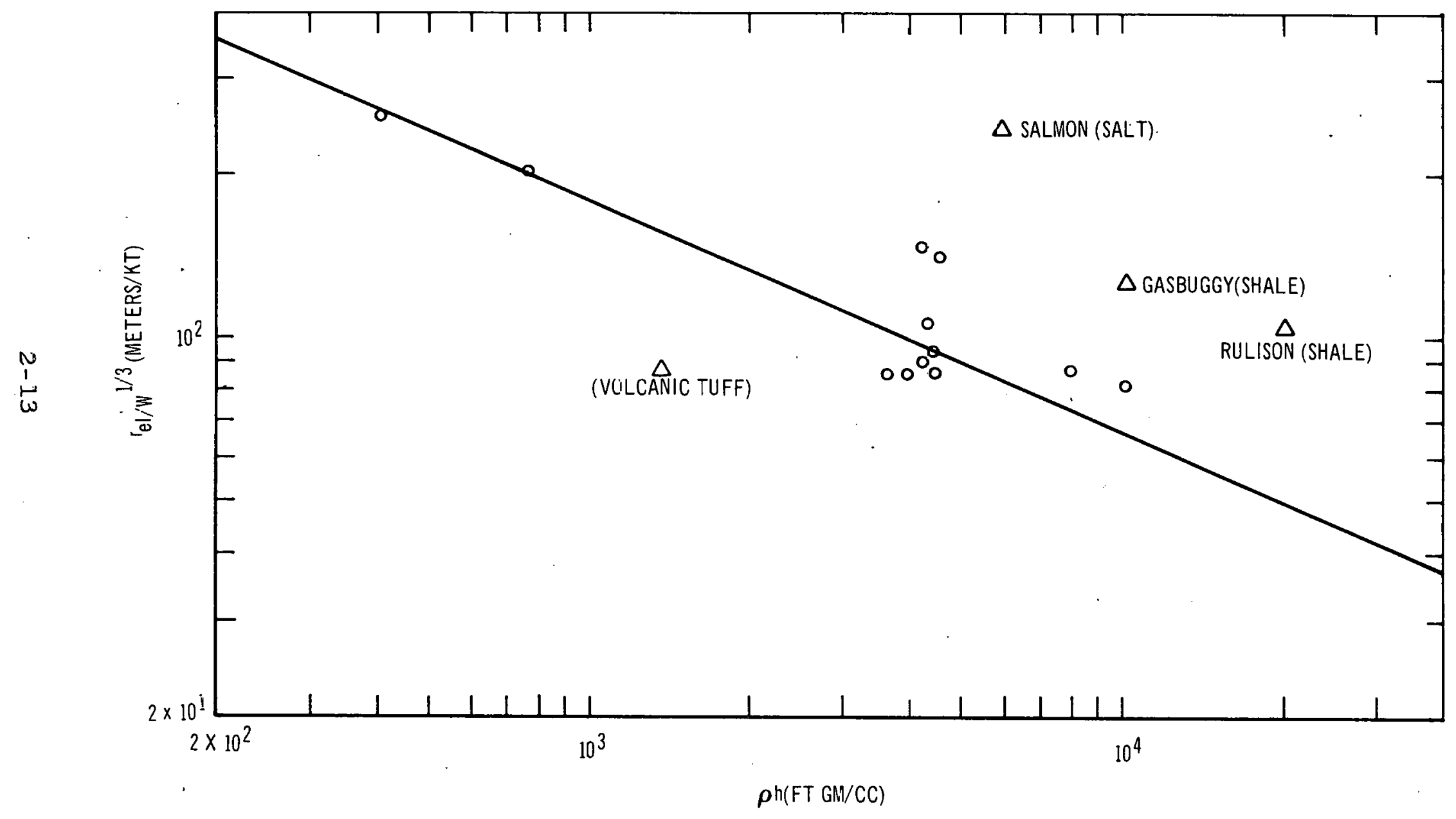

Figure 2-4. Plot of Elastic Radius/Cube Root of Yield Versus Density Times Depth of Burial for Various Shots (Circles are Events Detonated in Tuff or Rhyolite) 
Also shown are shots deto nated in salt, shale, and high porosity volcanic tuff. The salt shot (Salmon) lies a factor of 2.8 higher than the base line which gives an $A_{\text {salt }} / A_{t-r}=(2.8)^{n}=12$. Two events in shale (Gasbuggy and Rulison) provide $A_{\text {shale }} \mathrm{A}_{\mathrm{t}-\mathrm{r}}=(2.0)^{\mathrm{n}}=5.3$. The volcanic tuff shot gives an $\mathrm{A}_{v t} / \mathrm{A}_{\mathrm{t}-\mathrm{r}}=(0.54)^{\mathrm{n}}=0.23$. Thus, the data of Figure 2-4 provide a base. for general scaling.

To summarize this chapter, complete scaling of the amplitude spectrum can be accomplished through equation (11) combined with an evaluation of $r_{e l}, \alpha, p_{o s}$, and $p_{o c} \cdot$ Other quantities are physical constants of the shot point medium. From Figure 2-4, a calibration elastic radius ( $r_{\text {el cal }}$ ) can be determined for shots in rhyolite and tuff and is defined as the elastic radius at $1 \mathrm{kt}$ and at $\rho \mathrm{h}=1 \mathrm{ft} \mathrm{gm} / \mathrm{cc} \cdot \mathrm{r}_{\mathrm{el}} \mathrm{cal}=$ 3280 meters. Thus, in general, for any event

$$
\frac{r_{e l}}{w^{1 / 3}}=\frac{r_{e l c a l}}{(\rho h)^{1 / n}}\left(\frac{A}{A_{\text {tuff-rhyolite }}}\right)^{1 / n}
$$

The remaining parameters utilized in equation (11) are:

$$
\begin{aligned}
a & =k \omega_{0} \\
\omega_{0} & =c / r_{e I}
\end{aligned}
$$




$$
\begin{aligned}
& \mathrm{p}_{\text {os }}=1.5 \mathrm{p}_{\mathrm{ob}} \\
& \mathrm{P}_{\mathrm{ob}}(\text { overburden pressure) }=\rho \mathrm{gh}
\end{aligned}
$$

and

$$
\mathrm{p}_{\mathrm{oc}}=\mathrm{d} \frac{4 \mu}{3}\left(\frac{\mathrm{r}_{\mathrm{c}}}{\mathrm{r}_{\mathrm{el}}}\right)^{3}
$$

where $d$ is the compaction factor representing the proportion of permanent displacement based on the incompressible model that is utilized. In the following chapters, it is found that a compaction factor appropriate for a tuff and rhyolite medium is 0.6 and for a shale-sandstone medium is 1.0. Empirical determinations of the cavity radius as a function of yield, depth of burial, and medium are presented in the next chapter. Chapter 3 will also describe some of the applications of the scaling theory. 
CHAPTER 3

APPLICATIONS OF THEORY

The seismic spectrum scaling theory derived in the last chapter will now be applied to empirical observations. The first item of interest is the form of the scaling scheme for events buried at typical depths of burial, i.e., at a scaled depth (SD) of about 400 where $S D=h / w^{1 / 3}(h$ in feet and $W$ in $\mathrm{kt}$ ). In order to accomplish this a calibration elastic radius is needed. For shots in rhyolite and tuff rel cal (the elastic radius at $1 \mathrm{kt}$ and $\rho \mathrm{h}=1 \mathrm{ft} \mathrm{gm} / \mathrm{cc}$ ) can be tained from Figure $2-4$ as 3280 meters. Cavity radii as a function of yield, depth of burial, and medium properties have been studied statistically by Closmann (1969). His correlation equation is

$$
r_{0}=21.0 w^{0.306} E^{0.514} \rho^{-0.244} \mu^{-0.576} h^{-0.161}
$$

where $r_{c}$ is cavity radius in meters, $W$ is yield in $k t, E$ and $\mu$ are the Young's and shear moduli, respectively, in megabars, 0 is overburden density in $\mathrm{gm} / \mathrm{cc}$, and $\mathrm{h}$ is depth of burial in meters. Extending the analysis to hiyhei yields gives the relation . 


$$
r_{c}=16.3 w^{0.29}\left(E^{0.62} \rho^{-0.24} \mu^{-0.67}\right) h^{-0.11}(25) *
$$

Average values of the factor $\left(E^{0.62} \rho^{-0.24} \mu^{-0.67}\right)$ are: granite, 1.513; salt, 1.721; rhyolite, 1.758; tuff, 1.927; alluvium, 1.761. To attain low frequency scaling consistent with the seismic data from tuff and rhyolite events, permanent displacements smaller than those predicted by the incompressible material model are necessary. This reduction is probably due to compaction which should increase with porosity. A compaction factor (d) of 0.6 is used.

Scaling is, in general, frequency, yield, and medium dependent. For shots in tuff and rhyolite around $200 \mathrm{kt}$ at a set scaled depth of 400 , yield scaling exponents, as a function of period (1/frequency) are plotted in Figure 3-1. The frequency or period ( $p)$ dependent yield exponents $(n(p))$ are obtained from the relation

$$
\left|\frac{\hat{z}_{1}}{\hat{z}_{2}}\right|=\left(\frac{\bar{w}_{1}}{\bar{w}_{2}}\right)^{n(p)}
$$

where the amplitude spectra ratio on the left is obtained from equation (II) for similar medium scaling. The theoretical

*Private communication from H.C. Heard and F.J. Ackerman of Lawrence Radiation Laboratory. 


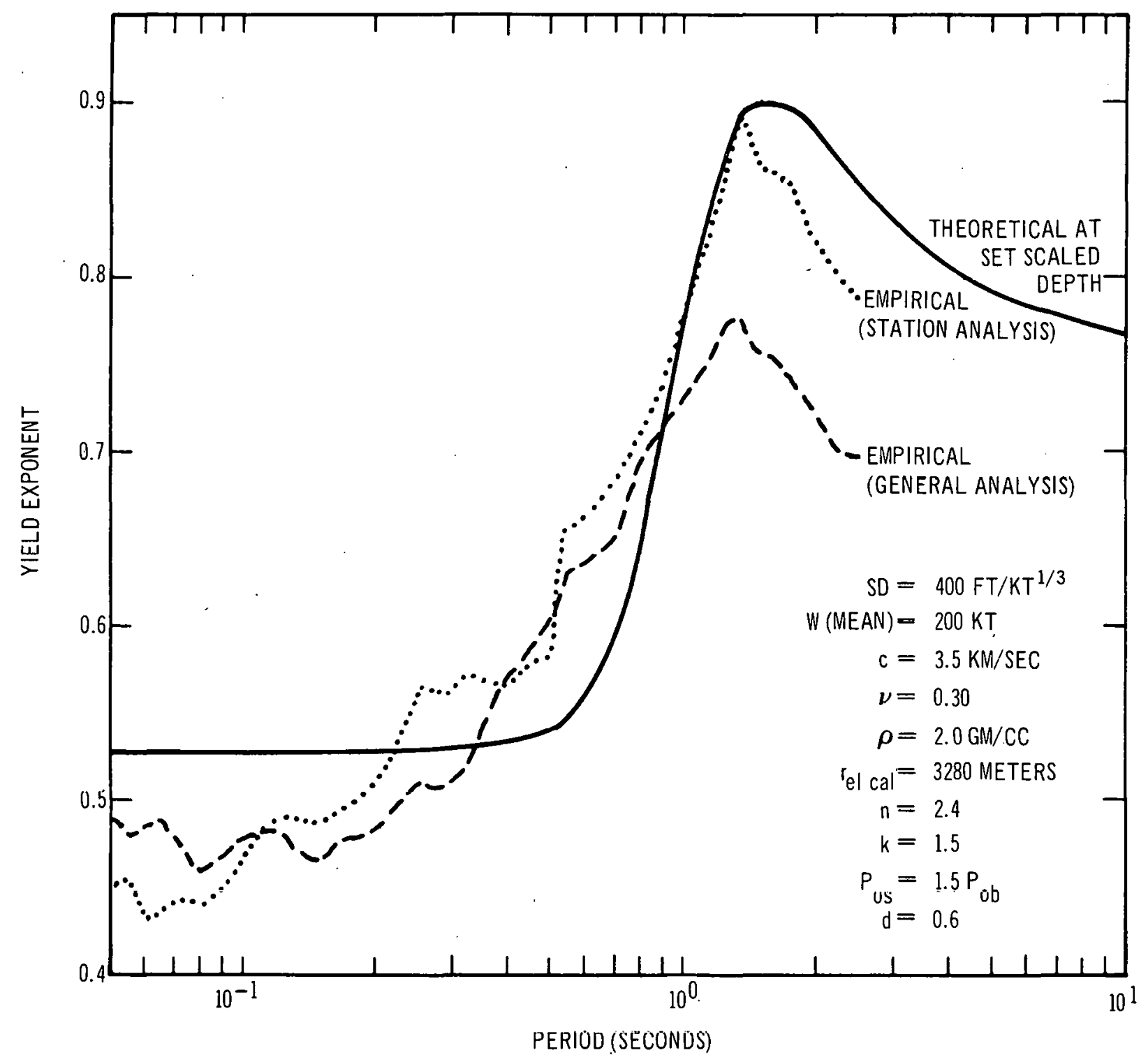

Figure 3-1. Comparison of Theoretical and Empirical Yield Scaling Exponents 
exponents are compared to exponents derived from a statistical analysis of PSRV (pseudo-relative velocity spectrum which Jensche (1967) and Hudson (1962) have shown to be similar to the Fourier amplitude spectrum) data from Pahute Mesa events in which distance and yield are independent variables (Lynch, 1969 ), and an average. station analysis at eight well instrumented stations in which yield alone is the independent variable (Mueller, 1970). The exponent curve illustrates the observed phenomenon of decreasing dominant frequency with increasing yield for typical contained events...

In order to simulate the empirical study more closely, the same least-squares criterion and averaging process is calculated analytically in the following way. First, theoretical amplitudes (relative) are computed as a function of period for each event included in the statistical sample for a given station, using the parameters and scaling relations defined above. The analytically derived amplitudes at each period are then fit to an equation of the form $A=K W^{n}$ using the least-squares criterion. The process is repeated for each of the eight recording stations under consideration and the yield exponents are averaged as a function of period. The results of these computations are shown in Figure $3-2$. It 


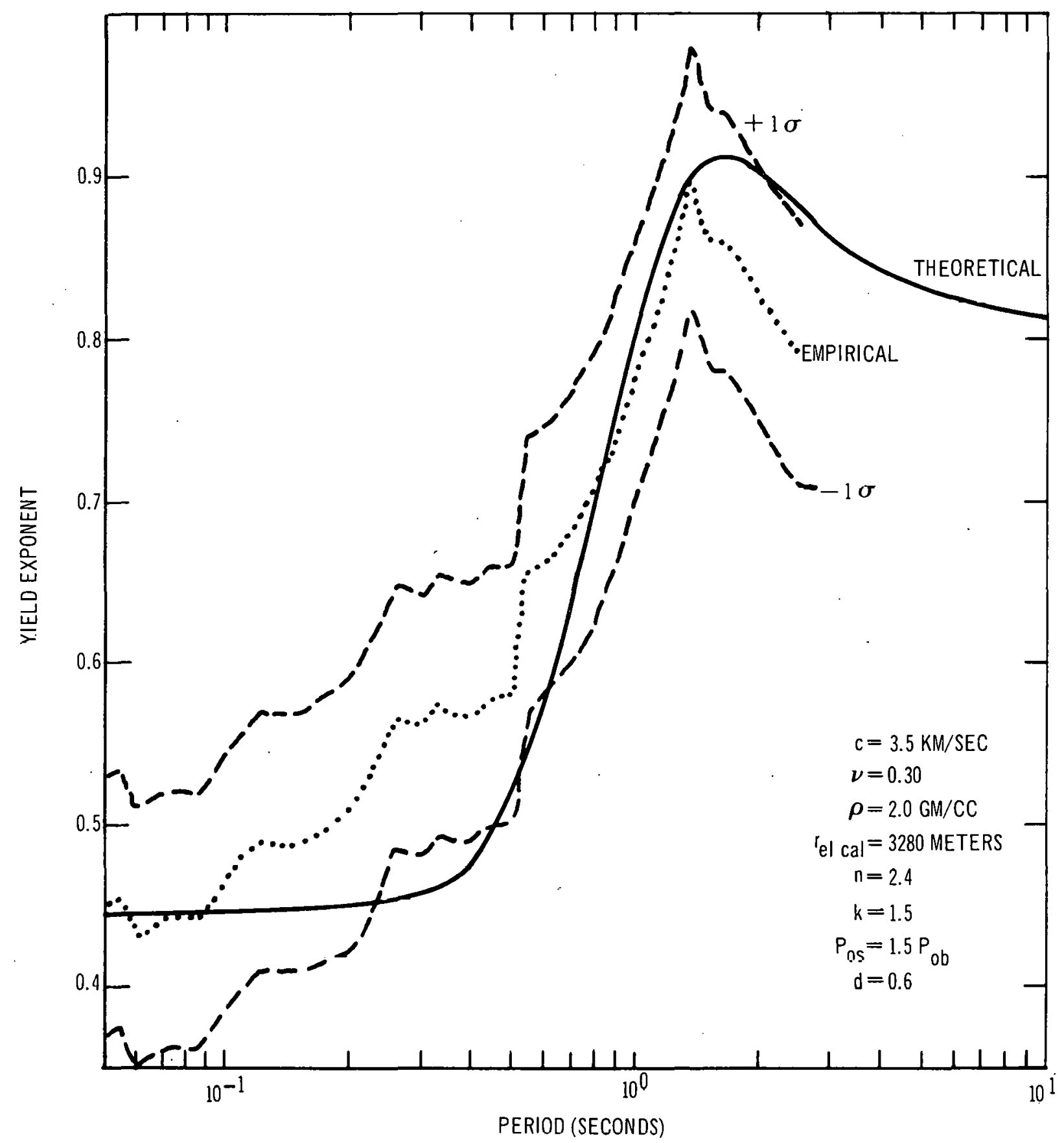

Figure 3-2. Comparison of Theoretical and Empirical Yield Scaling Exponents Derived from Eight Single Station PSRV Analysis 
can be seen that the theoretically derived yield exponents are in good agreement with those obtained from the measured data. The one sigma levels of the experimental data are also shown in the figure.

Complete amplitude spectra scaling of contained events detonated in tuff or rhyolite around $200 \mathrm{kt}$ and 2,330 feet depth of burial is described by Figures 3-3 and 3-4. Figure 3-3 gives yield scaling exponents as a function of period for events detonated at a set depth of burial. It predicts a shifting of the dominant energy to lower frequencies or longer periods as the yield increases. This curve also illustrates the result produced by simple cube-root yield scaling of the elastic radius, since, from equation (17), the elastic radius in a particular medium at a constant depth of burial is simply proportional to the cube-root of the yield. This result deviates considerably from: Figures $3-1$ and $3-2$ which indicates that simple cube-root yield scaling of the elastic radius, independent of depth of burial, is not appropriate. Figure 3-4 gives depth of burial scaling exponents as a function of period for events detonated at a set yield. It predicts a shifting of the dominant energy to higher frequencies or shorter periods as the depth of burial increases. Thus, complete 


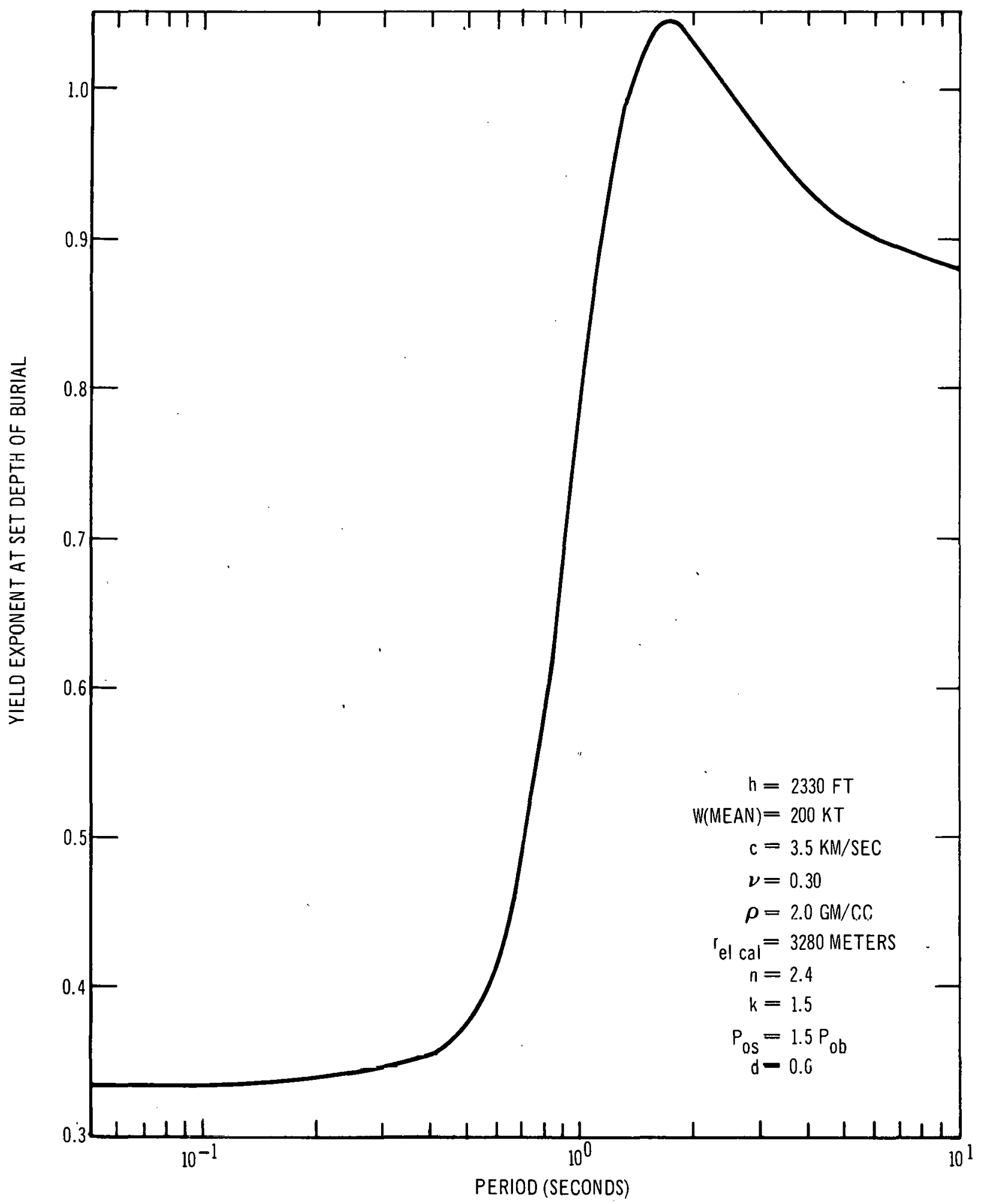

Figure 3-3. Theoretical Yield Scaling Exponents for Set Depth of Burial Events 


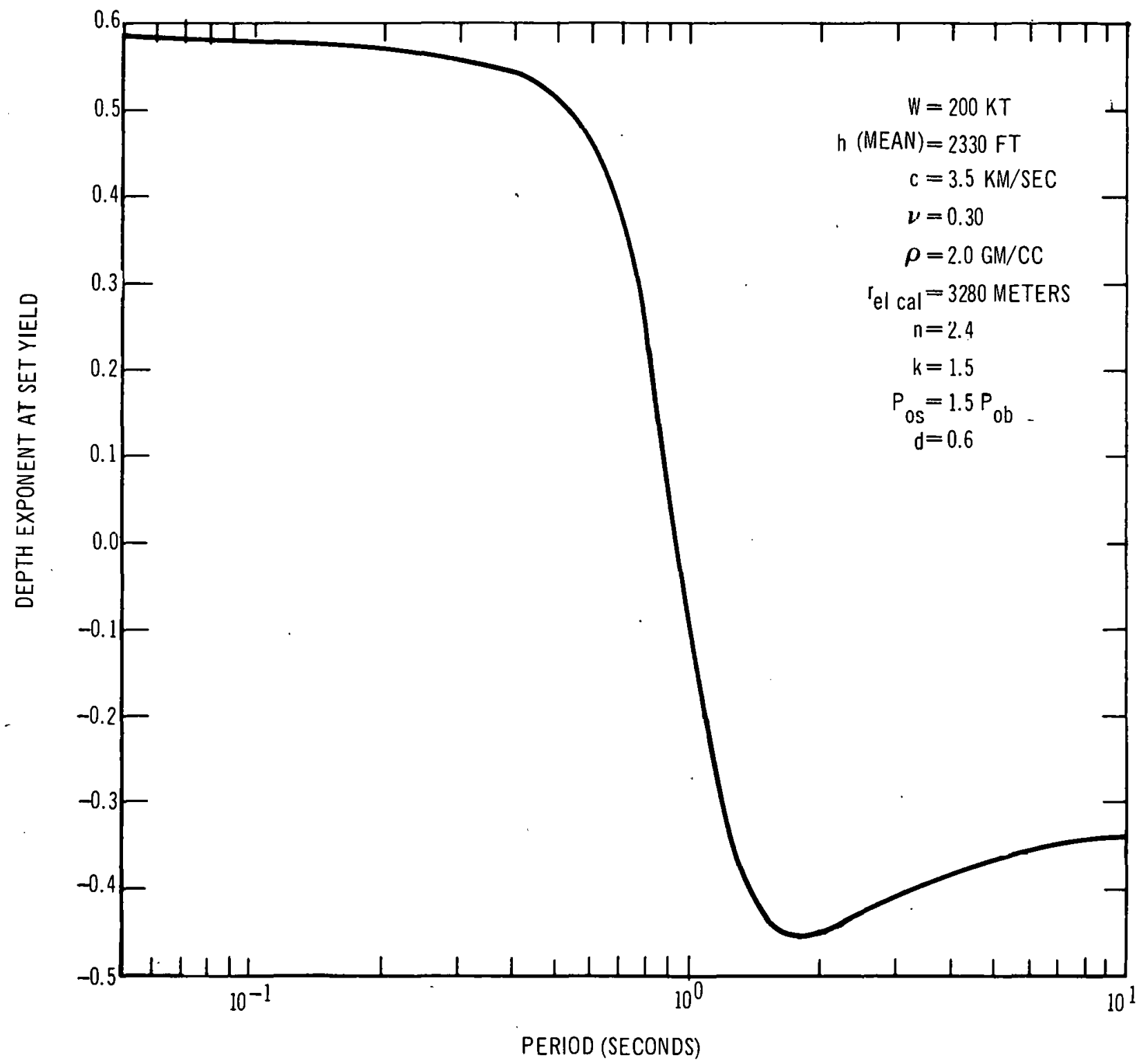

Figure 3-4. Theoretical Depth Scaling Exponents for Set Yield Events 
scaling from one event to another in tuff or rhyolite around $200 \mathrm{kt}$ and 2,330 feet depth of burial is given by

$$
\left|\frac{\hat{z}_{1}}{\hat{z}_{2}}\right|=\left(\frac{w_{1}}{w_{2}}\right)^{s(p)}\left(\frac{h_{1}}{h_{2}}\right)^{t(p)}
$$

where the yield exponent at a set depth of burial $(s(p))$ is given by Figure $3-3$ and the depth of burial exponent at a set yield $(t(p))$ is given by Figure $3-4$.

The next application of the scaling scheme is to individual events. Events buried at relatively small and large depths of burial are compared to events buried at typical contained depths of burial.

First, the excavation events Cabriolet and Schooner are compared to the contained events Knickerbocker and Rex. This analysis indicates that the final cavity radius for excavation events at a scaled depth of 120 is approximately 0.75 the cavity radius that would be predicted for contained events at that scaled depth. The smaller cavity radius is obviously due to the cavity breaking the surface. Utilizing this information, the PSRV from the contained events are theoretically scaled to the yields and depths of burial of the excavation events at several stations (Figures 3-5 through 3-8). 


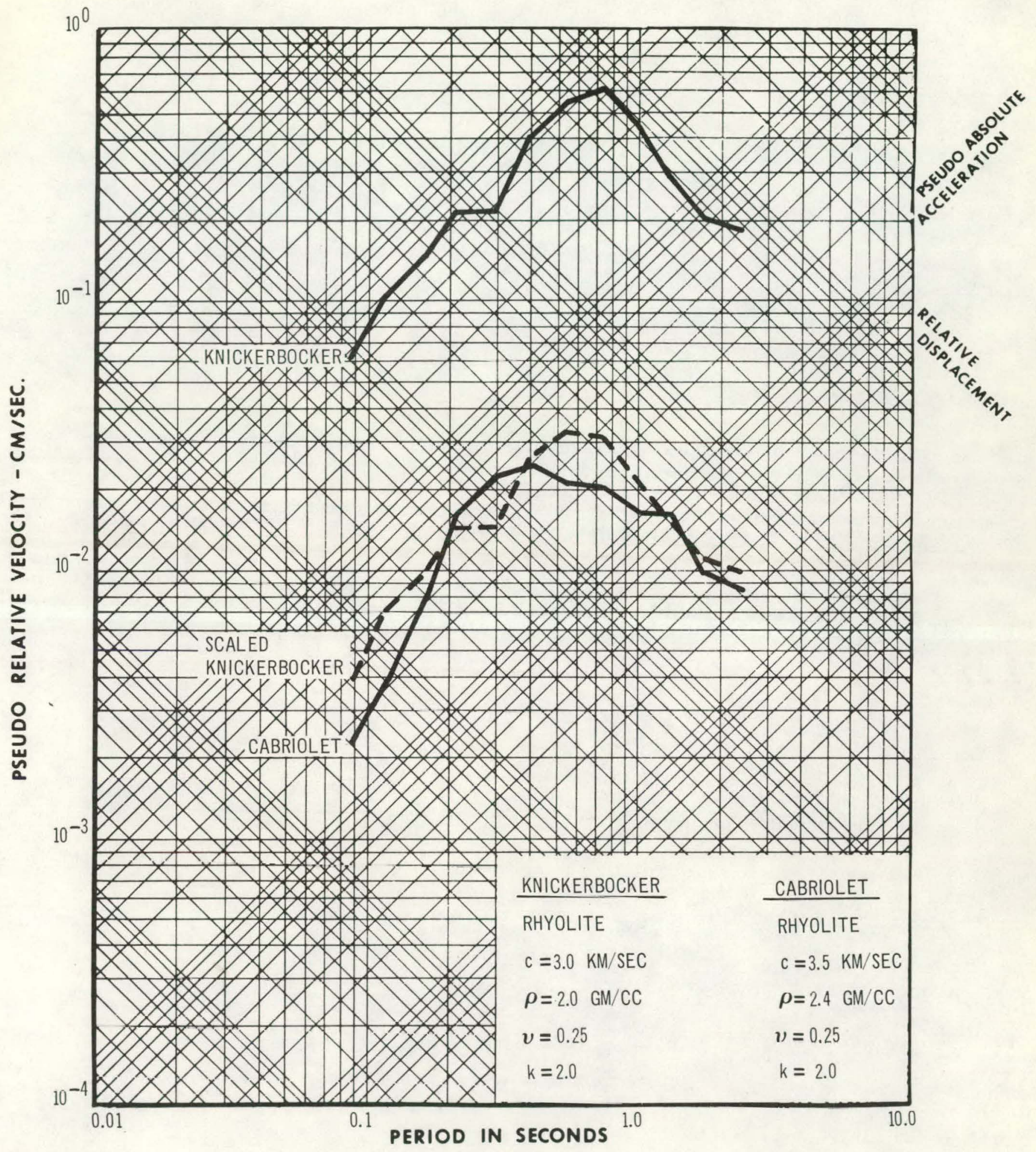

Figure 3-5. Comparison of Knickerbocker, Scaled Knickerbocker and Cabriolet PSRV (5\% Damping) at Alamo (Horizontal Component) 


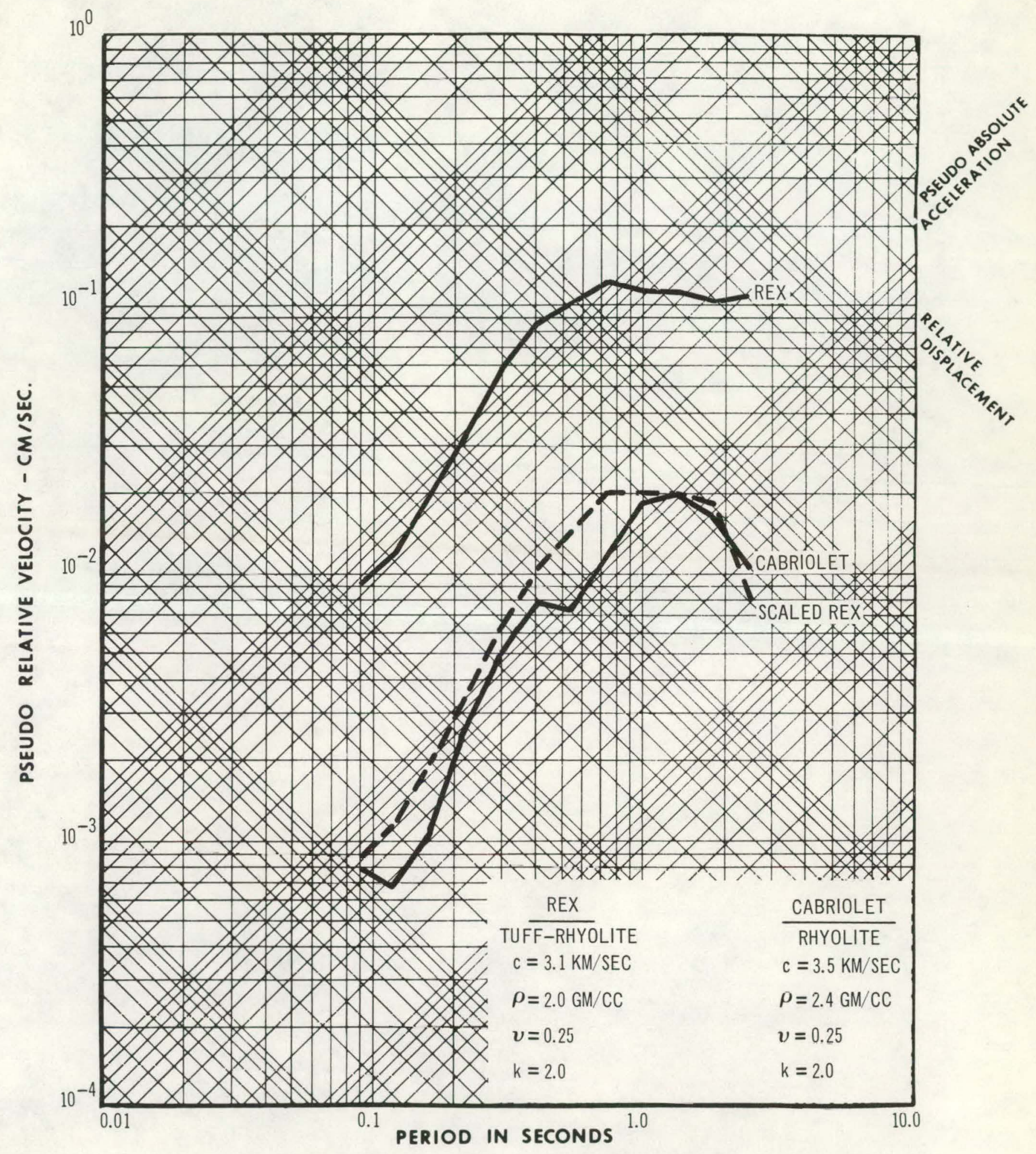

Figure 3-6. Comparison of Rex, Scaled Rex and Cabriolet PSRV (5\% Damping) at SE-6 (Horizontal Component) 


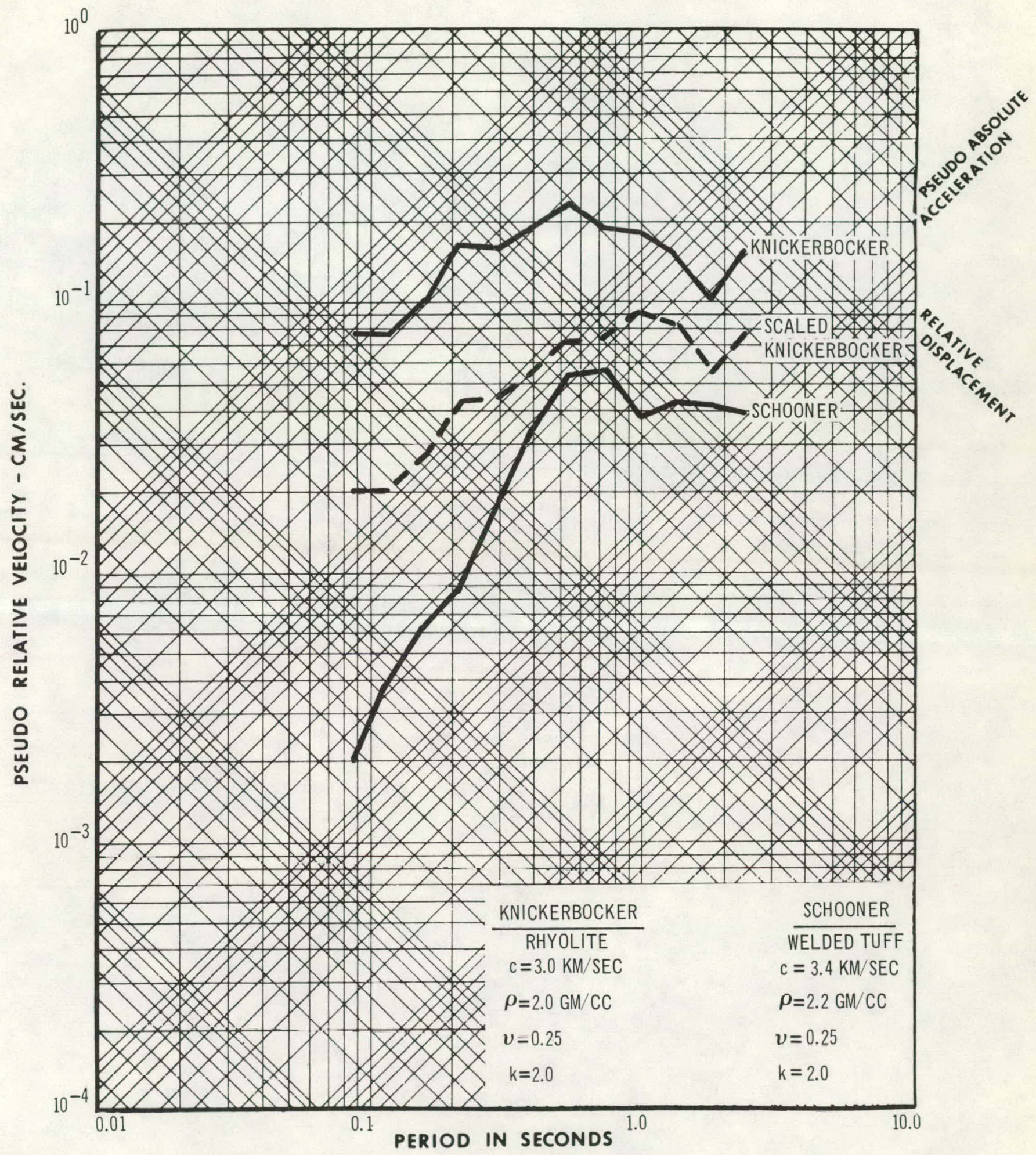

Figure 3-7. Comparison of Knickerbocker, Scaled Knickerbocker and Schooner PSRV (5\% Damping) at Tonopah Church (Horizontal Component) 


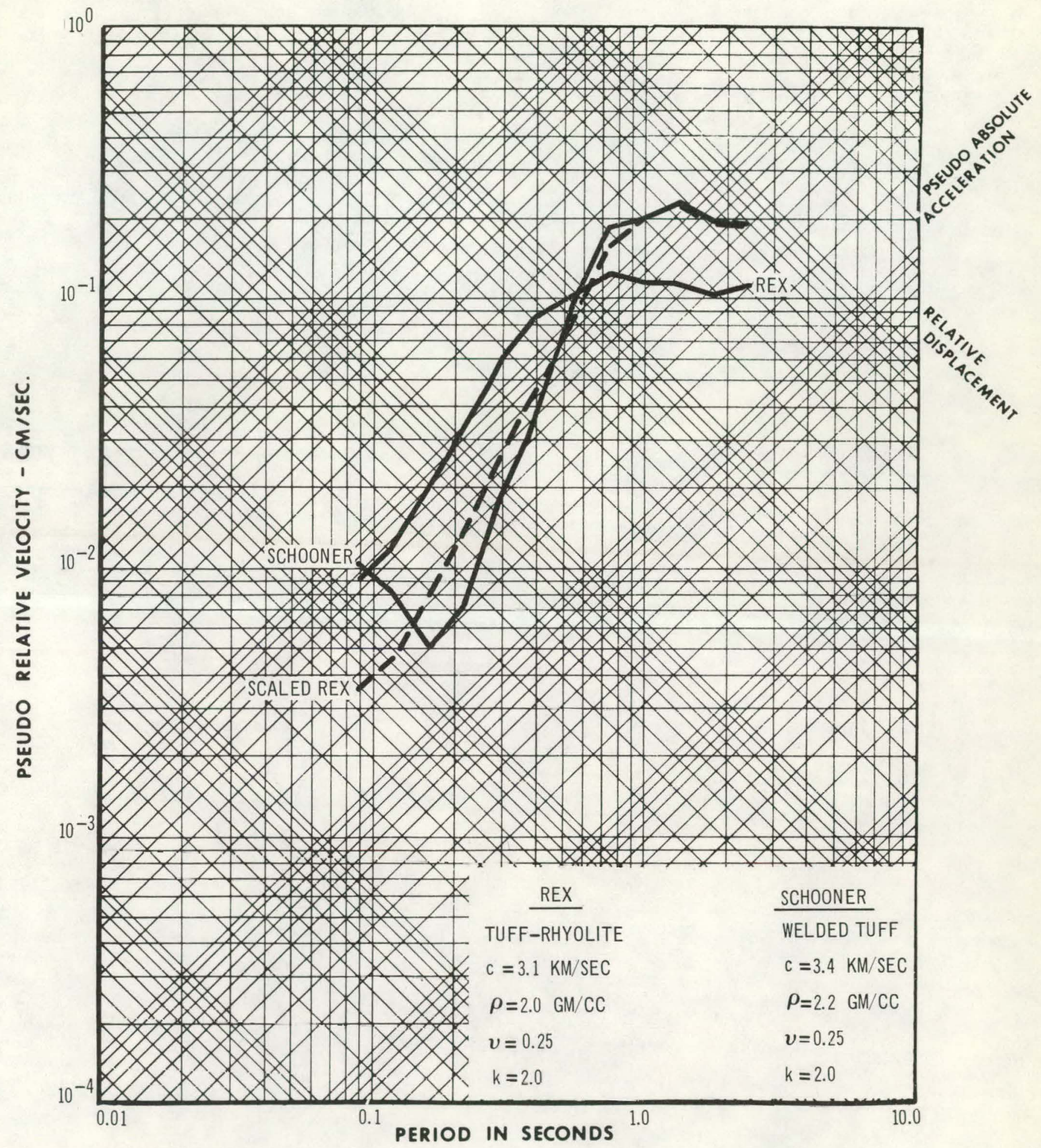

Figure 3-8. Comparison of Rex, Scaled Rex and Schooner PSRV (5\% Damping) at SE-6 (Horizontal Component) 
To illustrate the difference in spectral character between contained and excavation events, the Knickerbocker and Rex events are shown in Figures 3-9 and 3-10, scaled to the same yields as the excavation events Cabriolet and Schooner, but at contained depths of burial corresponding to a scaled depth of 400. All the source parameters of the scaled contained events are the same as the excavation events except for depth of burial. Comparison of the spectra from the two different types of events shows that a contained event generates a higher level of motion; more enriched at higher frequencies or shorter periods than an excavation event.

The results presented above indicate that the assumption of spherical symmetry still holds for excavation events detonated at a scaled depth of 120 or greater. It is unclear how shallow an event must be or how severe of an inhomogeneity must exist before the assumption of spherical symmetry in a homogeneous medium at the source breaks down.

The next test of the scaling scheme is on a low yield, overburied Event A at station SE-6 where it recorded at a good signal to noise ratio throughout the frequency range of interest. Figure 3-11 illustrates the spectra from Event A, Greeley (a high yield event), and the result of scaling Event A to Greeley. 


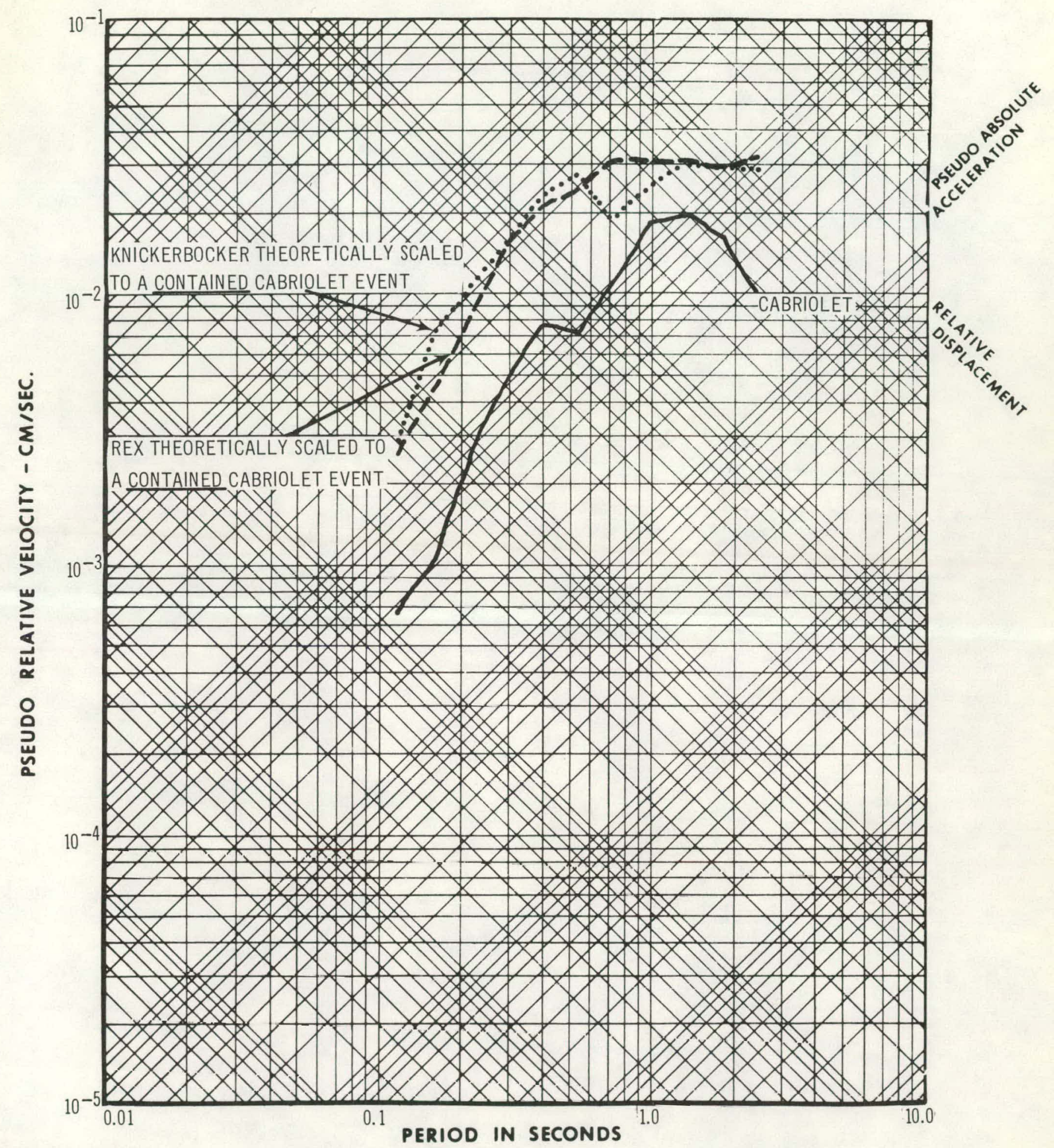

Figure 3-9. Comparison of PSRV (5\% Damping) from Cabriolet with PSRV from Knickerbocker and Rex Theoretically Scaled to Equivalent Physical Parameters Except at a Contained Depth of Burial $(\mathrm{SD}=400)$, Station SE-6, Horizontal Component 


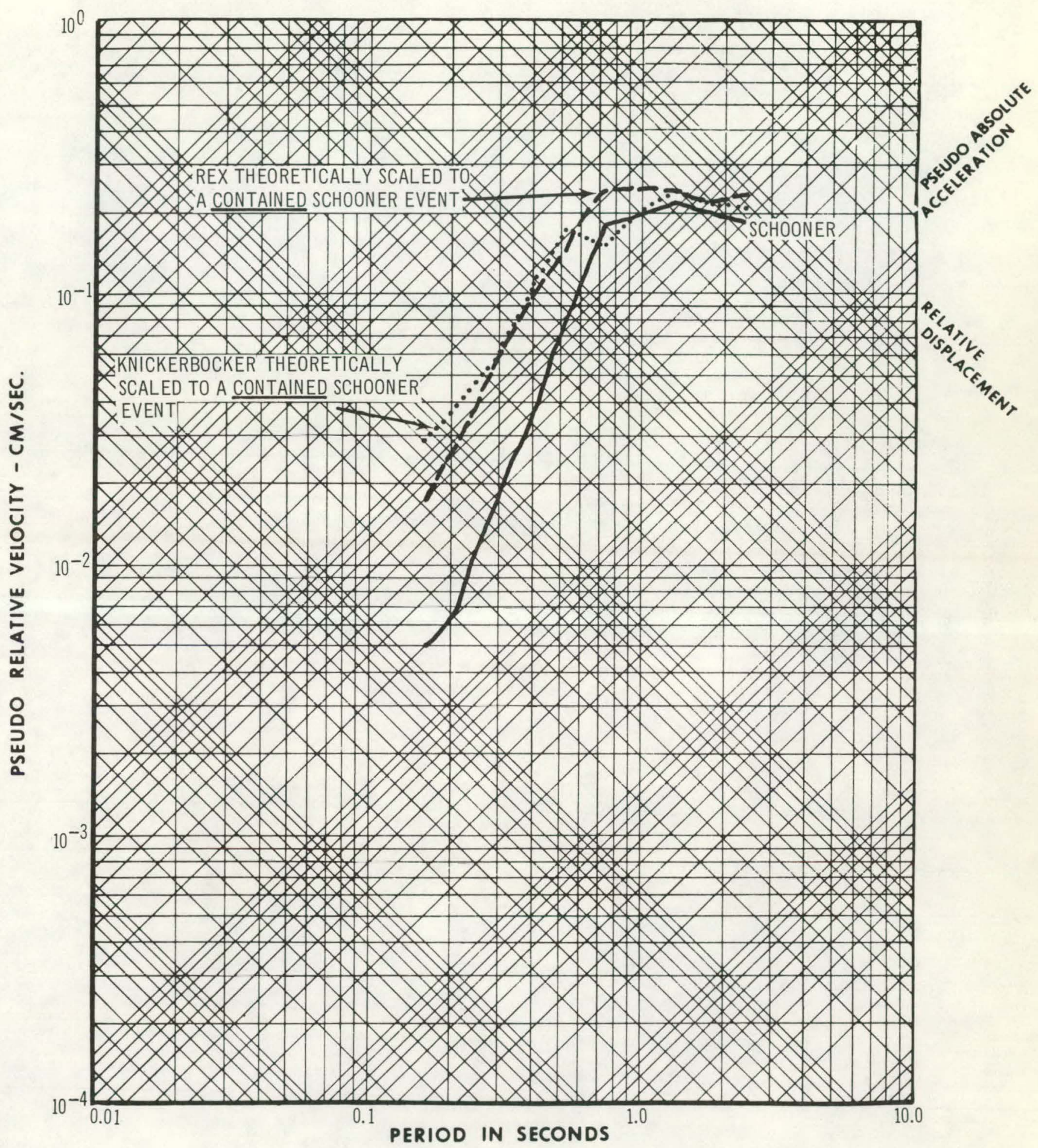

m-gure 3-10. Comparison of PSRV (5\% Damping) from Schooner with PSRV from Knickerbocker and Rex Theoretically Scaled to Equivalent Physical Parameters Except at a Contained Depth of Burial $(S D=400)$, Station SE-6, Horizontal Component 


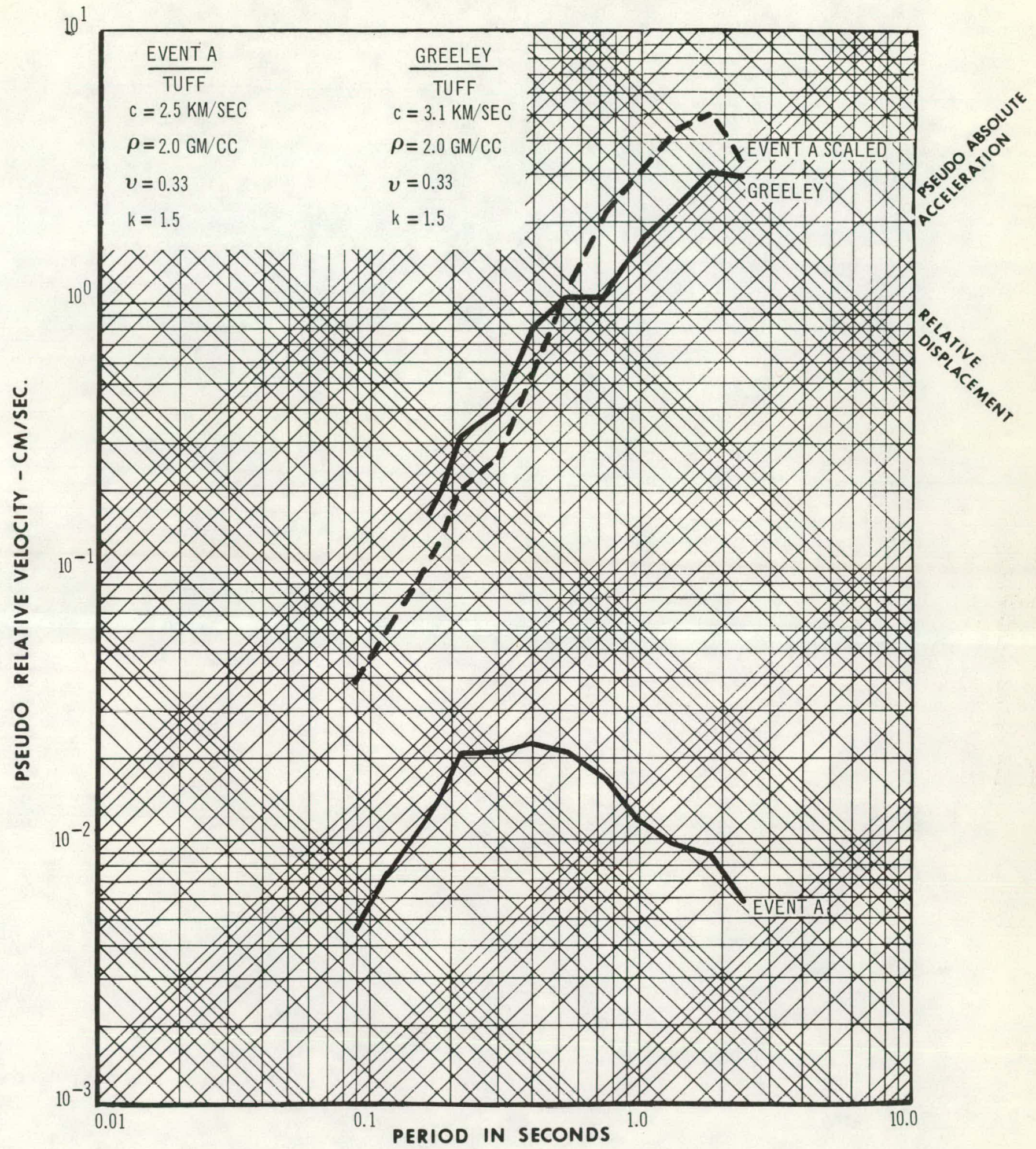

Figure 3-11. Comparison of Event A, Event A Scaled and Greeley PSRV (5\% Damping) at SE-6 (Horizontal Component) 
This result demonstrates the effect of scaling events with widely different yields detonated at essentially the same depth of burial. The dominant energy is observed to shift to lower frequencies or longer periods at the higher yield. It is also of interest to investigate events detonated at drastically different depths of burial. Figure 3-12 compares the spectra from Event A, Cabriolet, and the result of scaling Event A to Cabriolet. The dominant energy is observed to shift to higher frequencies or shorter periods at the greater depth of burial.

The results of this chapter demonstrate the validity of the general scaling theory presented in Chapter 2. 


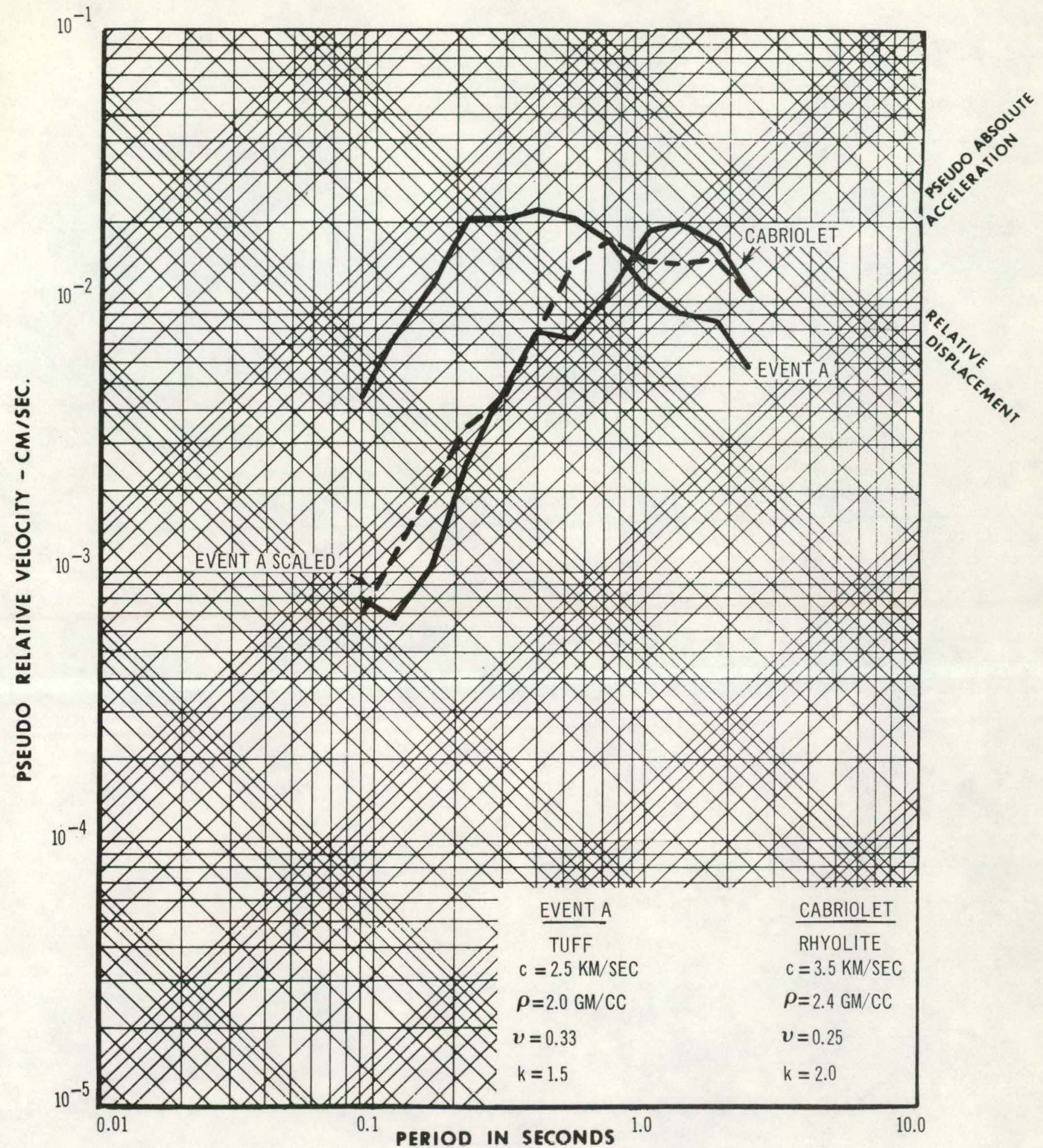

Figure 3-12. Comparison of Event A, Event A Scaled and Cabriolet PSRV (5\% Damping) at SE-6 (Horizontal Component) 


\section{CHAPTER 4}

\section{EXTENSION OF THE SPECTRUM SCALING THEORY}

TO PEAK GROUND MOTION SCALING

In this chapter, the seismic spectrum scaling theory is extended to peak particle motions. Jensche (1967) and Hudson (1962) have demonstrated empirically that PSRV and Fourier amplitude spectra are similar. Moreover, Jensche (1967) has shown that the asymptotic limit of the relative displacement spectrum should approach the peak displacement as fre- quency goes to zero and the asymptotic limit of the pseudo abśolute acceleration spectrum should approach the peak acceleration as frequency goes to infinity. The low frequency limit of the scaling equation ( 1 i) is

$$
\left|\frac{z_{1}}{z_{2}}\right|_{\omega \rightarrow 0}=\left(\frac{\mathrm{r}_{\mathrm{e}} \mathrm{I}_{1}}{\mathrm{r}_{\mathrm{e} I_{2}}}\right)^{3} \frac{\mu_{2}}{\mu_{1}} \frac{\mathrm{Poc}_{1}}{\mathrm{p}_{\mathrm{oc}}}
$$

Utilizing equation (15) we have

$$
\left|\frac{z_{1}}{z_{2}}\right|_{\omega \rightarrow 0}=\frac{d_{1}}{d_{2}}\left(\frac{r_{c_{1}}}{r_{s_{2}}}\right)^{3}
$$

where d (compaction factor) represents the proportion of permanent displacement based on the incompressible material model. This parameter is a function of the compaction processes. 
Assuming that the compaction factor remains constant for events in the same medium, the low frequency limit becomes

$$
\left|\frac{z_{1}}{z_{2}}\right|_{\omega \rightarrow 0}=\left(\frac{{ }^{r_{c_{1}}}}{r_{c_{2}}}\right)^{3}
$$

Utilizing equation (25) for the cavity radius gives

$$
\left|\frac{z_{1}}{Z_{2}}\right|_{\omega \rightarrow 0}=\left(\frac{w_{1}}{w_{2}}\right)^{0.87}\left(\frac{h_{2}}{h_{1}}\right)^{0.33}
$$

for events detonated in the same medium.

The high frequency limit of equation (11.) is

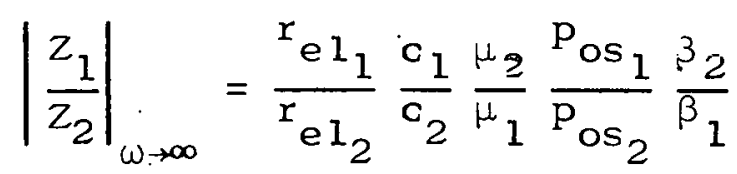

Taking $p_{\rho s}$ to be proportional to $\rho g h$ and using equation (19), we get

$$
\left|\frac{Z_{1}}{Z_{2}}\right|_{\omega \rightarrow \infty}=\left(\frac{A_{1}}{A_{2}}\right)^{1 / n}\left(\frac{\rho_{1} h_{1}}{\partial_{2} h_{2}}\right)^{(1-1 / n)}\left(\frac{W_{1}}{w_{2}}\right)^{1 / 3} \frac{c_{1} \mu_{2} \beta_{2}}{c_{2} \mu_{1} \beta_{1}}
$$

Using the relations $\beta=c^{2} / 4 c_{s}^{2}$ and $\mu=c_{s}^{2} \rho$, where $c_{s}$ is the shear velocity, reduce equation (31) to 


$$
\left|\frac{Z_{1}}{Z_{2}}\right|_{\omega \rightarrow \infty}=\left(\frac{A_{1}}{A_{2}}\right)^{1 / n}\left(\frac{h_{1}}{h_{2}}\right)^{(l-1 / n)}\left(\frac{\rho_{1}}{\rho_{2}}\right)^{(-1 / n)} \frac{c_{2}}{c_{1}}\left(\frac{W_{1}}{W_{2}}\right)^{1 / 3}
$$

For scaling events detonated in the same medium, equation (32) becomes

$$
\left|\frac{Z_{1}}{Z_{2}}\right|_{\omega \rightarrow \infty}=\left(\frac{h_{1}}{h_{2}}\right)^{(1-1 / n)}\left(\frac{W_{1}}{w_{2}}\right)^{1 / 3}
$$

with $\mathrm{n}$ determined to be about 2.4 .

It is of interest to investigate the peak displacement and peak acceleration yield scaling exponents for events at a set scaled depth. In this case, $h=S D^{*} W^{1 / 3}$ and the yield exponents corresponding to equations (29) and (33) become 0.76 for peak displacements and 0.53 for peak accelerations. These exponents are to be compared to the 99 event statistical average exponents determined by Murphy and Lahoud (1969). They found peak displacement yield scaling exponents between 0.76 and 0.85 and peak acceleration yield soaling exponents between 0.61 and 0.66 .

We will now investigate the consequences of peak motion scaling as applicd to the Gasbuggy event (yield $26 \mathrm{kt}$; depth of burial, 4240 $f t$, detonated in a shale, sandstone medium. The regression equationsderived from the 9.9 event statistical analysis (Murphy and 
Lahoud (1969)) are used as a basis. The data sample consists principally of ground motion data from shots detonated in tuff, alluvium and rhyolite. Thus, medium constants assoiated with this sample can only be approximated. The average compressional velocity, density and scaled depth were taken to be $3.5 \mathrm{~km} / \mathrm{sec}, 2.0 \mathrm{gm} / \mathrm{cc}$ and 400 respectively while the constants for the Gasbuggy event are $4.75 \mathrm{~km} / \mathrm{sec}, 2.4$ $\mathrm{gm} / \mathrm{cc}$ and 1432 respectively. Figure $4-1$ shows the peak acceleration regression equations for hard rock and alluvium stations at $26 \mathrm{kt}$, and the Gasbuggy data. Using the above constants in equation (32) together with an $n$ of 2.4 and the observation from Figure $2-4$ that $\left(A_{\text {Gasbuggy }} / A_{B a s e}\right)^{1 / n}=2.0$ determines the "medium and depth of burial factor" to be 2.9 . The scaled lines are also shown in Figure 4-1 for comparison.

The $\mathrm{A}_{\mathrm{B} a s e}$, used above for the 99 event sample, was developed for shot's detonated in tuff and rhyolite. This value of A for the 99 event sample is probably somewhat high since the sample included a considerable number of events detonated in alluvium. The conclusion is that medium scaling should probably be somewhat greater.

Equation (27) is used for the peak displacement scaling. Gasbuggy's cavity radius is $80 \mathrm{ft}$ and a typical $26 \mathrm{kt}$ event detonated in tuff or alluvium would produce a cavity radius of about $132 \mathrm{ft}$. Using a compaction factor of 1.0 for Gasbuggy (no compaction) and 0.6 for the 99 event sample gives a 


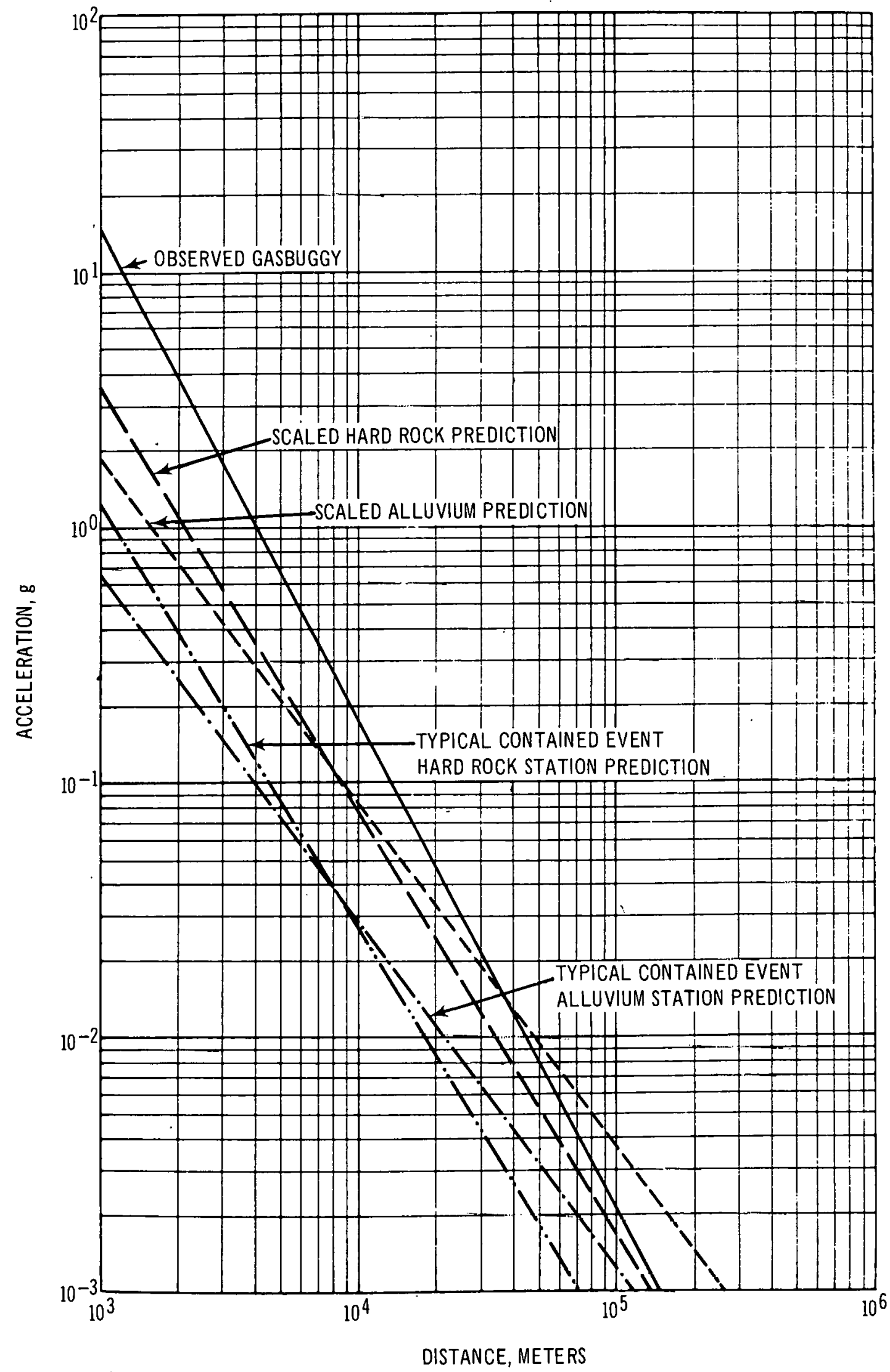

Figure 4-1. Comparison of Observed Gasbuggy and Predicted Peak Accelerations at Hard Rock and A.T luvium Stations 
low frequency scaling factor of 0.37 . Figure $4-2$ shows the peak displacement Iines. It appears that the displacement scaling might be too great. A possible explanation may exist in the compaction factor (d) used for the 99 event sample, which is based on events detonated in tuff and rhyolite, and/or a breakdown in the relation between the Fourier amplitude spectrum and the PSRV spectrum at the asymptotic Iimit. A compaction factor of 0.24 for the 99 event sample would produce correspondence of the Gasbuggy and the scaled hard rock prediction at $50 \mathrm{~km}$.

Although the method of peak acceleration and displacement scaling described above is reasonable, it neglects the effects of the earth's low-pass filtering characteristics. It does not allow the differential attenuation effects of the earth to play a role in shaping the distance slope of the peak motions.

Peak velocities can be handled in a more effective manner. Since the peak in the frequency domain can be associated with the peak velocity in the time domain, scaling can be accomplished directly from the peak spectral ratio. This procedure allows the differential attenuation effects of the earth to affect the scaling. To illustrate this procedure, PSRV derived from NTS 


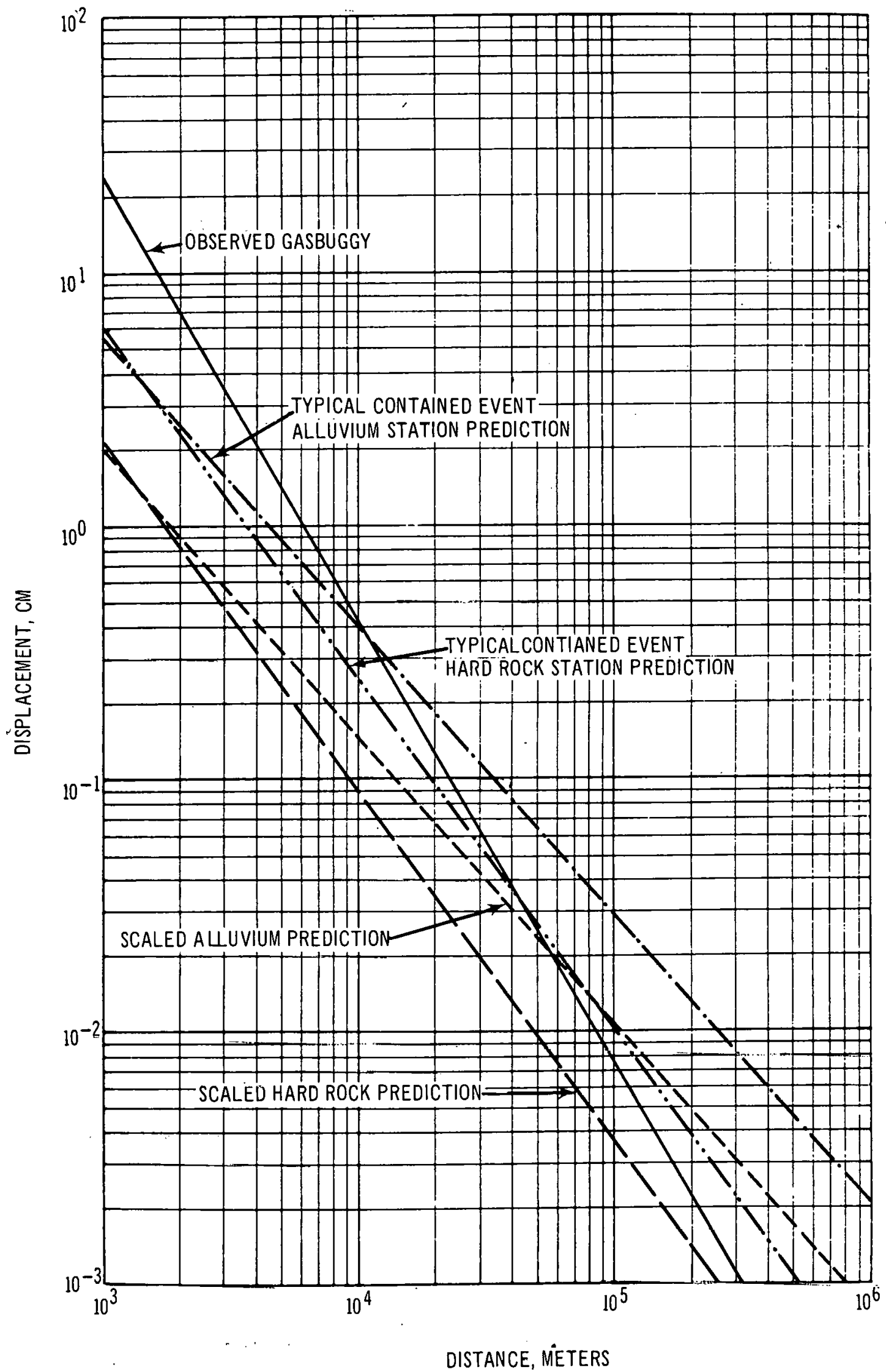

Figure 4-2. Comparison of Observed Gasbuggy and Predicted Peak Farticle Displacement.s at Hard Rock and Alluvium Stations 
experience (Mueller, 1970) were scaled to the Gasbuggy source parameters at station:distances of $10 \mathrm{~km}$ and $80 \mathrm{~km}$. Figures 4-3 and 4-4 show the results. The Gasbuggy PSRV is based on a regression analysis of the measured spectra (Foote, Hays, and Klepinger, 1969). Although an exact correspondence between NTS and Gasbuggy PSRV should not be expected due to the fact that the data samples are different, the relation between scaled and unscaled NTS-PSRV should display the desired results. The scaled spectra show the expected enrichment at higher frequencies due to the overburied nature and shot point medium characteristics of Gasbuggy. From Figures 4-3 and 4-4, the peak velocity scaling factors are: at $10 \mathrm{~km}, 2.3$; at 80 $\mathrm{km}, 1.7$. Application of these factors to the peak velocity data is shown in Figure 4-5.

Figures $4-6$ and $4-7$ show a comparison of peak accelerations and displacements scaled from Gasbuggy to Rulison (yield, $40 \mathrm{kt}$; depth of burial, 8443 feet; shale-saidstonc medium) using the empirical yield exponents derived by Murphy and Lahoud (1969). and the theoretical equations (29) and (33). Better agreement. is obtained through the theoretical scaling.

The results shown in this chapter illustrate that the procedure developed for peak motion scaling is reasonable and Fairly accurate in most cases. 


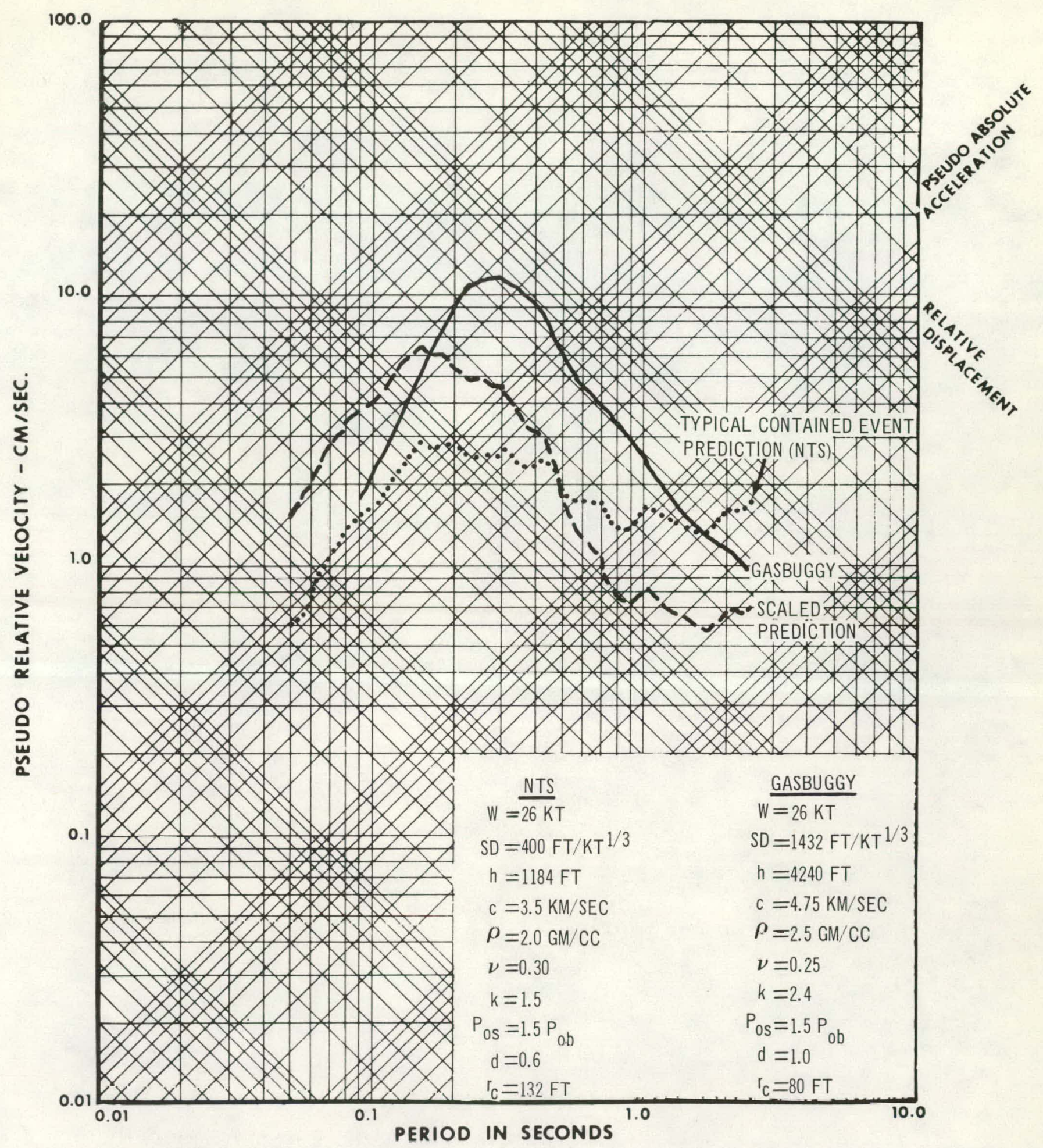

Figure 4-3. Comparison of Gasbuggy and Predicted PSRV (5\% Damping) at $10 \mathrm{~km}$ 


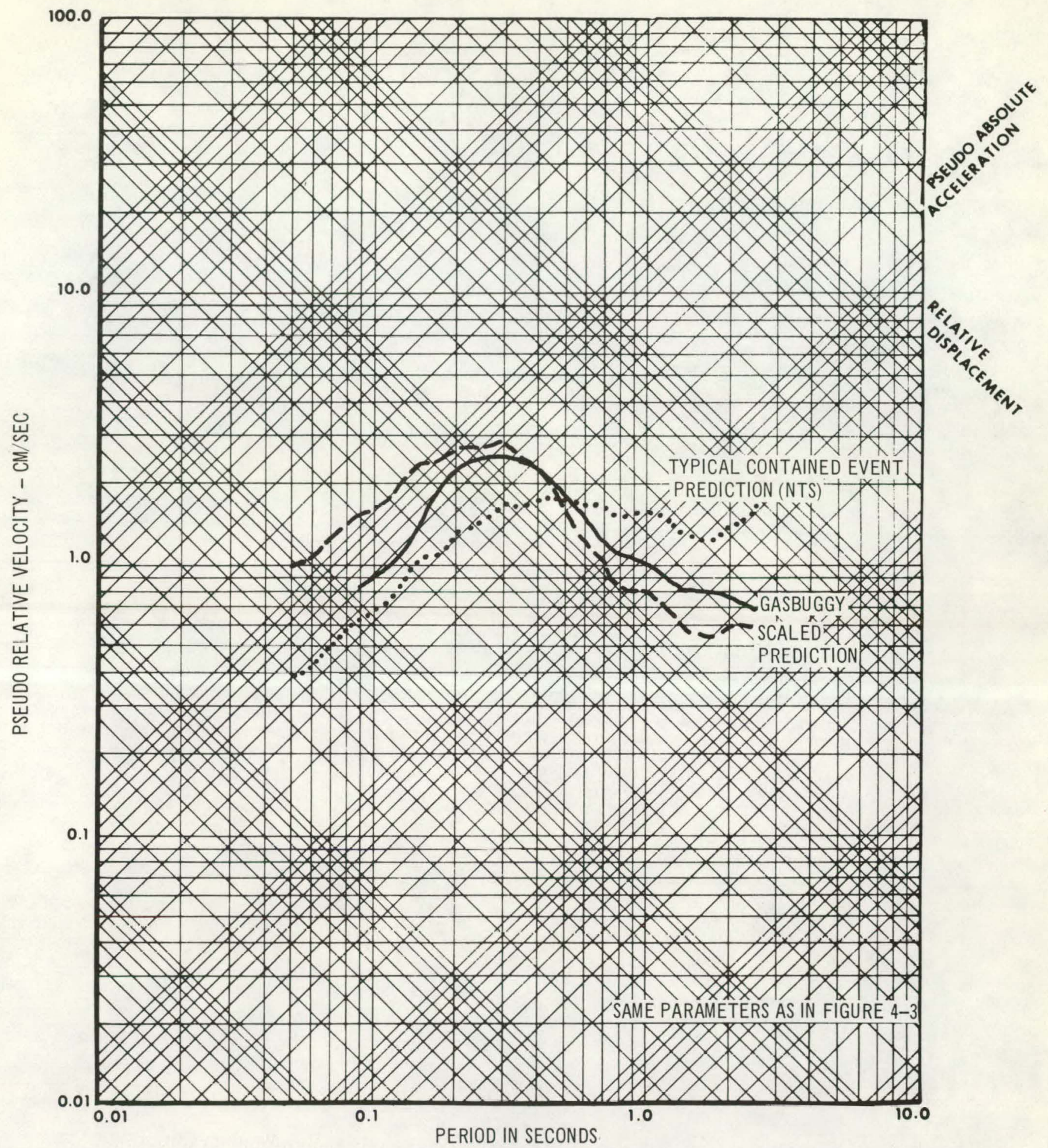

Figure 4-4. Comparison of Gasbuggy and Predicted PSRV (5\% Damping) at $80 \mathrm{~km}$ 


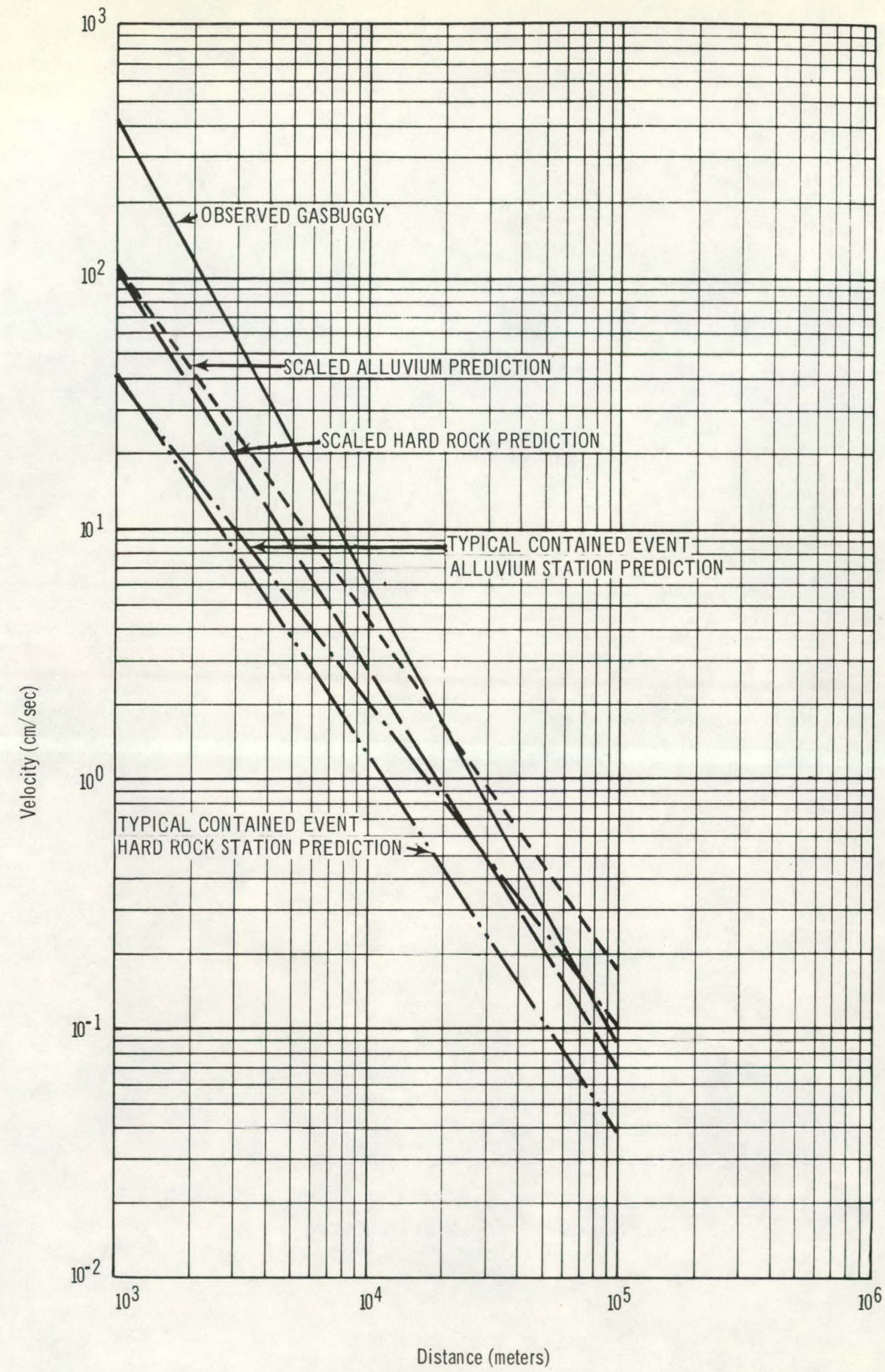

Figure 4-5. Comparison of Observed Gasbuggy and Predicted Peak Particle Velocities at Hard Rock and Alluvium Stations 4-11 


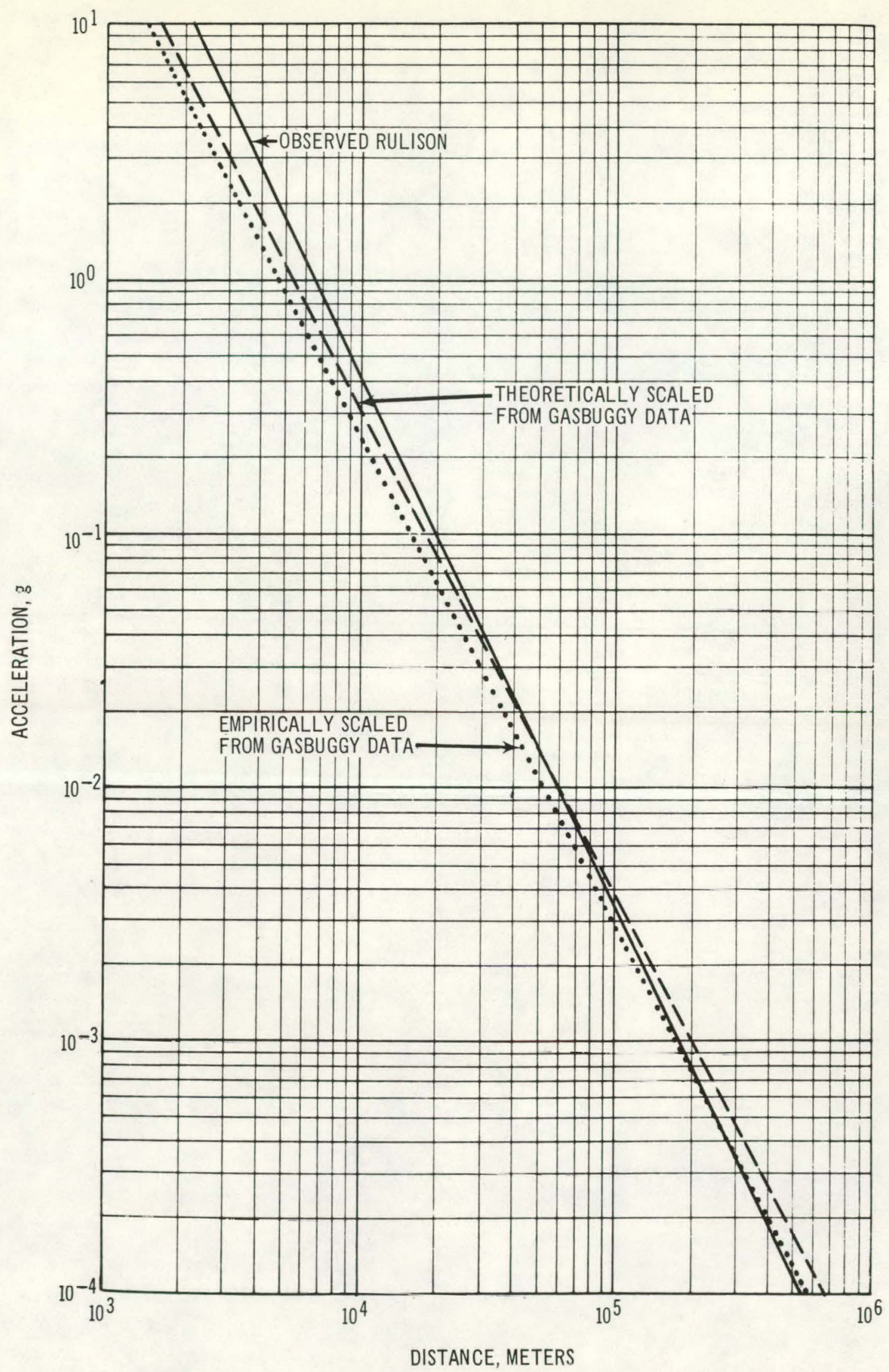

Figure 4-6. Comparison of Observed Peak Particle Accelerations with the Accelerations Theoretically and Empirically Scaled from Gasbuggy 


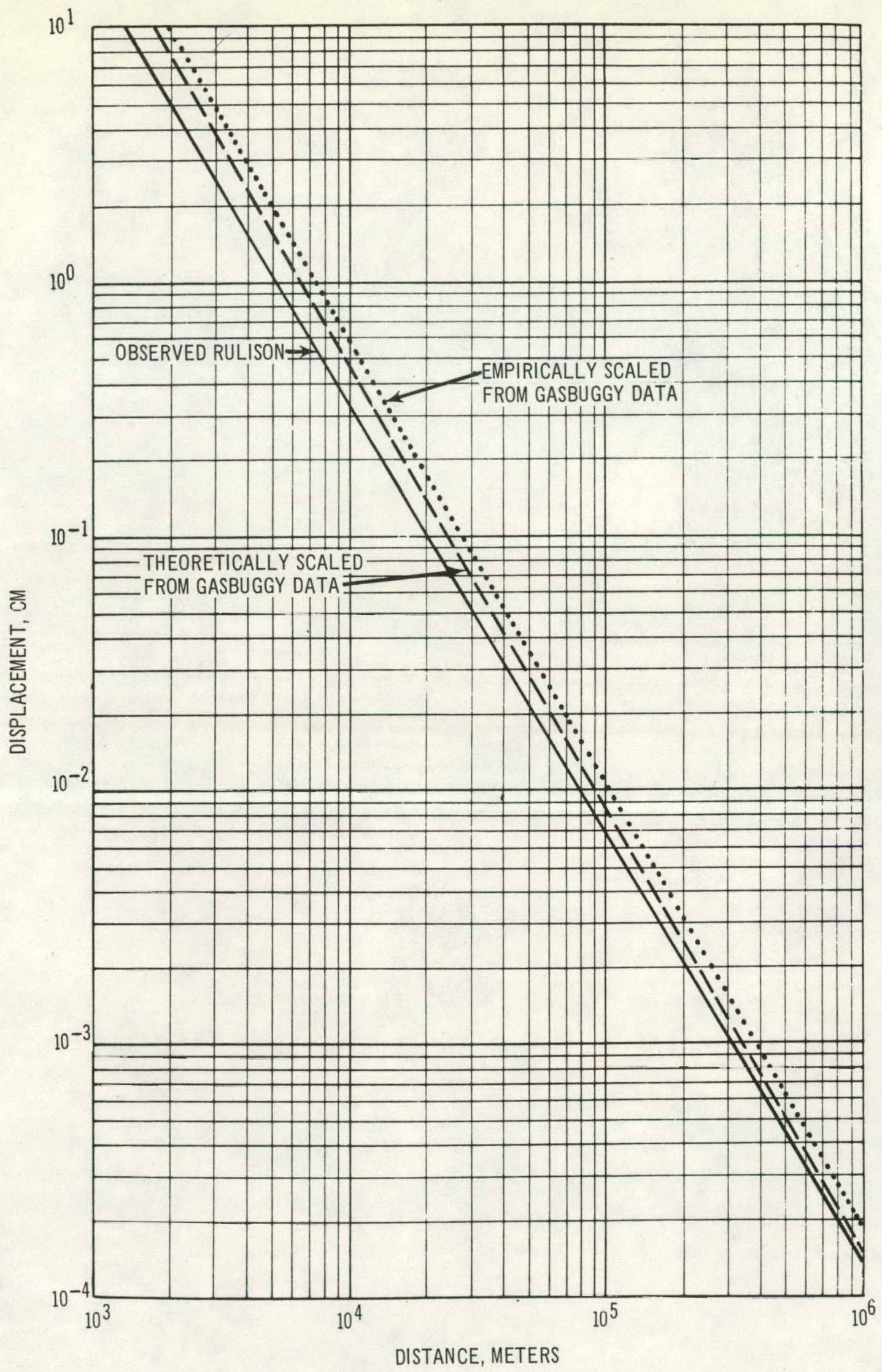

Figure 4-7. Comparison of Observed Rulison Peak Particle Displacements with the Displacements Theoretically and Empirically Scaled from Gasbuggy

$$
4-13
$$




\section{CHAPTER 5}

SUMMARY AND RECOMMENDATIONS

A general theory for scaling the ground motions resulting from underground nuclear detonations is developed from the solution of the wave equation with appropriate boundary conditions. This theory shows that yield, device depth of burial, and the emplacement medium play a significant role in the scaling of seismic signals. The theory predicts:

- an increase in the dominant frequency and ground accelerations with an increase in device depth of burial (overburied detonations).

- a decrease in the dominant frequency and ground accelerations with a decrease in device depth of burial (nuclear excavation detonations).

- frequency dependent yield exponents which range from 0.90 at low $f$ requencies to 0.45 at high $f$ requencies for typical contained events.

Results based on the general scaling theory were compared with the experimental results observed from various nuclear detonations. This comparison shows good agreement between theory and experimental results. Specific comparisons and a summary uf the results are as follows: 
- Comparison of Theoretical and Experimental Yield Scaling Exponents - The theoretical yield scaling exponents derived on the basis of a mean $200 \mathrm{kt}$ nuclear detonation in tuff and rhyolite at a scaled.depth of 400 agree with the yield scaling exponents derived from a statistical analysis of PSRV data measured from Pahute Mesa detonations (Lynch, 1969).

- Comparison of Spectra from Cratering Detonations and Contained Detonations - PSRV spectra observed from Cabriolet and Schooner (cratering detonations) were compared with the PSRV spectra from Knickerbocker and Rex (contained detonations). The comparison was effected by scaling the spectra of the contained events for differences in yield, depth of burial, and medium. The result substantiates the fact that the high frequency content of a cratering detonation is considerably reduced relative to that of an equivalent yield contained detonation.

- Comparison of Spectra from Overburied Detonations and Typical Contained Detonations - Spectra from Event A(overburied) were scaled for yield and depth in order to be compared with spectra from the Greeley (typical contained) event. This comparison shows that the spectra of overburied detonations are characterized by a higher dominant frequency and a higher level of peak particle accelerations. These phenomena are related to the fact that overburial causes the elastic radius to be smaller. The smaller elastic radius, which may be thought of as representing a resonant cavity whose characteristic frequency is determined by its radius, results in a higher dominant frequency of generation for the elastic waves. An effect of the higher frequency content is to enhance the peak particle accelerations.

- Scaling of Peak Particle Vector Ground Mutions Yield scaling exponents for peak particle vector accelerations $(0.53)$ and displacements $(0.76)$ were derived on the basis of theoretical considerations. The yield scaling exponents derived in this manner compare well with the yield scaling exponents determined from a statistical analysis of peak accelerations and displacements observed from 99 underground nuclear detonations. The peak particle vector acceleration, velocity, and displacement predictions, based on NTS experience, 
were scaled for the Gasbuggy depth of burial, yield and medium differences and compared with the observed data. These data show that these predictions, based on sraling theory and NTS data sample, agree with the observed Gasbuggy data. Basic differences are due in part to the differential attenuation effects of the earth's low-pass filtering action with distance. Similar results are obtained for the Rulison data.

The scaling theory developed in this report can be applied to predict the PSRV spectra and peak ground motions for special purpose nuclear detonations. Events for which this theory has been applied include Gasbuggy (Foote et al, 1969), Rulison (Weetman et al, 1969; Foote, et al, 1970), Schooner (Hays, et al, 1969), and Cabriolet (Klepinger and Mueller, 1969).

Further development of the scaling theory is recommended. Additional research into the effect of the physical properties of various emplacement sites is required to develop the theory fully for application to detonations in new test sites characterized by properties significantly different from those at Nevada Test Site. Additional verification of the theory will be acquired by comparing the theory and experimental. results obtained from future detonalions such as. Wasp, Wagon wheel and Sturtevant. 


\section{REFERENCES}

Closmann, P...., 1969, On the Prediction of Cavity Radius Produced by an Underground Nuclear Explosion, J.G.R., 74 3935-3939.

Foote, R.Q., W.W. Hays, and R:W. Klepinger, 1969, Analyses of Ground Motions and Close-In Physical Effects - Gasbuggy Event, PNE-1010, Clearinghouse for Fed. Scientific \& Tech. Infor., NBS, Springfield, Virginia.

Foote, R.Q., C.R. Cassity, P.C. Loux, L.J. O'Brien, F.W. Power, W.W. Hays, R.D. Lynch, F.R. Perchalski, C.T. Spiker, and A.P. Whipple, 1970, Analysis of Ground Motions and CloseIn Physical Effects, Rulison Event, Environmental Research Corporation, NVO-1163-206, AEC.

Hays, W.W., R.A. Mueller, and C.T. Spiker, 1969, Schooner Event, A Contribution to the Analysis of Seismic Data From Cratering and Contained Events, Environmental Research Corporation, NVO-1163-188, AEC.

Hudson, D.E., 1962, Some Problems in the Application of Spectrum Techniques to Strong-Motion Earthquake Analysis, BSSA 52, 417-430.

Jenschke, V.A., R.W. Clough and J. Penzien, 1967, Characteristics of Strong Ground Motions, Report to Ernest 0 . Radiation Laboratory, Livermore, California.

Klepinger, R.W., and R.A. Mueller, 1969, Analysis of Ground Motion from Cralering and Contained Events, Cabriolet, PNE-958, Clearinghouse for Fed. Sci. \& Tech. Infor., NBS, Springfield, Va.

Latter, A.L., E.A. Martinelli and E. Teller, 1959, Seismic Scaling Law for Underground Explosions,. Physics of Flulds 2, 280-282.

Lynch, R.D., 1969, Response Spectra for Pahute Mesa Nuclear Events, BSSA 59, \#6, 2295-2309.

Mueller, R.A., 1969, Seismic Energy Efficiency of Underground Nuclear Detonations, BSSA. 59, \#6, 2311-2323. 
Mueller, R.A., 1970, Prediction of Seismic Motion from Contained and Excavation Nuclear Detonations. Proceedings: Engineering with Nuclear Explosives, American Nur. ear Society, 1051-1068.

Murphy, J.R. and J.A. Lahoud, 1969, Analysis of Seismic Peak Amplitudes from Underground Nuclear Explosions, BSSA 59, \#6, 2325-2341.

Perret, W.R., 1965, Free-Field Ground Motion Studies in Granite (U), Operation Nougat, Shot Hard Hat; Sandia Corporation, POR-1803.

Perret, W.R., 1968, Free-Field Particle Motion from a Nuclear Explosion in Salt, Part I, Project Dribble, Salmon Event, Sandia Laboratory, VUP-3012.

Sauer, F.M., 1964, Ground Motion from Underground Nuclear Explosions, Nuclear Geoplosics, DASA-1285(IV).

Sharpe, J.A., 1942, The Production of Elastic Waves by Explosion Pressures, I. Theory and Empirical Obser: vations, Geophysics 18, 144-154.

Weart, W.D., 1965, Free-Field Earth Motion and Spalling Measurements in Granite, Project Shoal, Sandia Corporation:' VUP-2001.

Weetman, B.G. and Others, 1969, Predictions of Seismic Motion and Close-In Effects. - Rulison Event, Environmental Research Corporation, NVO-1163-180, AEC. 
Distribution - NVO-1i63-195

Dr. E.M. Douthett, AEC/NVOO, Las Vegas, Nevada (2 copies)

Mr. A.D. Thornbrough, AEC/NVOO, Las Vegas, Nevada ( 1 copy)

Technicn 1 . I.ibraxy, AEC/NVOO, Las Vegas, Nevada ( 1 copy)

Mr. R. Loux, AEC/NVOO; Las Vegas, Nevada ( 1 copy)

AEC/DOS, Hq., Washington, D.C. (I copy)

AEC/DPNE, Hq., Washington, D.C. ( 1 copy)

AEC/DMA, Hq., Washington, D.C. (2 copies)

DTIE, Oak Ridge, Tennessee (2 copies)

Mr. Richard Hamburger, AEC/DPNE, Hq., Washington, D.C. (1 copy)

Dr. G.H. Higgins, LRL, Livermore, California ( 1 copy)

Dr. H.L. Reynolds, LRL, Livermore, California ( 1 copy)

Dr. G.C. Werth, LRL, Livermore, California ( 1 copy)

Dr. J.W. Hadley, LRL, Livermore, California (2 copies)

Dr. F. Holzer, LRL, Livermore, California (1 copy)

Dr. W. E. Ogle, LASL, Los Alamos, New Mexico ( 1 copy)

Mr. R.W. Newman, LASL, Los Alamos, New Mexico ( 1 copy)

Dr. J.R. Banister, Sandia Corp., Albuqueruqe, New Mexico ( I copy)

Dr. M.L. Merritt, Sandia Corp., Albuqueruqe, New Mexico (1 copy)

Dr. W.D. Weart, Sandia Corp., Albuquerque, New Mexico (1 copy)

Technical Library, Sandia Corp., Albuquerque, New Mexico ( 1 copy)

Mr. T.F. Thompson, San Francisco, California ( 1 copy)

Dr. N.M. Newmark, Univ. of Illinois, Urbána, Illinois (l copy)

Mr. S.D. Wilson, Shannon \& Wilson, Inc., Seattle, Washington.(l copy)

Dr. D.U. Deere, Univ. of Illinois, Urbana, Illinois ( 1 copy)

Dr. L.S. Jacobsen, AEC, Los Angeles, California ( 1 copy)

Dr. G.B. Maxcy, University Station, Reno, Nevada ( 1 copy)

Mx́. 'L.G. vonLossberg, Sheppard T. Powell \& Associates, Baltimore,

Maryland ( 1 copy)

Dr. J.T. Wilson, Univ. of Michigan, Ann Arbor, Michigan (1 copy)

Dr. C. Kisslinger, St. Louis University; St. Louis, Missouri (l copy)

Dr. E.F. Cox, Benton Harbor, Michigan ( 1 copy)

Dr. J.A. Blume, Jolu A. Blume \& $\Lambda$ ssociates, San Francisco, California ( 2 copies)

Dr. W.S. Twenhofel, USGS, Denver, Colorado ( 1 copy)

Mr. P.L. Russel1, USBM, Denver, Colorado ( 1 copy)

Mr. W. Mickey, USC\&GS, Rockvilie, Maryland ( 1 copy)

Mr. K.W. King, USC\&GS, Las Vegas, Nevada (2 copies)

Dr. L.B. Werner, Isotopes, Inc., Palo Alto, California (2 copies).

Dr. R.H. Sproull, ARPA, Washington, D.C. ( 1 copy)

Dr. Benjamin Grote, TCD-B, DASA, Sandia Base, Albuquerque,

New Mexico ( 2 copies)

Environmental Research Corporation, Las Vegas, Nevada ( 3 copy)

It. Comdr. R. O. Johnstone, ACDA/WEC/Fo, Washington, D.C. ( 1 copy) 
This report was prepared as an account of Government sponsored work. Neither the United States, nor the Commission, nor any persun acting on behalf of the Commission:

A. Makes any warranty or representation, expressed or implied, with respect to the accuracy, completeness, or usefulness of the information contained in this report, or that the use of any information, apparatus, method, or process disclosed in this report may not infringe privately owned rights; or

B. Assumes any liabilities with respect to the use of, or for damages resulting from the use of any information, apparatus, method, or process disclosed in this report.

As used in the above, "persons acting on behalf of the Commission" includes any employee or contractor of the Commission, or employee of such contractor, to the extent that such employee or contractor of the Commission, or employee of such contractor prepares, disseminates, or provides access to, any information pursuant to his employment or contract with the Commission, or his employment with such contractor. 